\title{
A stability constrained adaptive alpha for gravitational search algorithm
}

${ }^{4}$ Department of Electronic and Electrical Engineering, University of Strathclyde, Glasgow, G1 1XW, United Kingdom

\section{Abstract:}

Gravitational search algorithm (GSA), a recent meta-heuristic algorithm inspired by Newton's law of gravity and mass interactions, shows good performance in various optimization problems. In GSA, the gravitational constant attenuation factor alpha $(\alpha)$ plays a vital role in convergence and the balance between exploration and exploitation. However, in GSA and most of its variants, all agents share the same $\alpha$ value without considering their evolutionary states, which has inevitably caused the premature convergence and imbalance of exploration and exploitation. In order to alleviate these drawbacks, in this paper, we propose a new variant of GSA, namely stability constrained adaptive alpha for GSA (SCAA). In SCAA, each agent's evolutionary state is estimated, which is then combined with the variation of the agent's position and fitness feedback to adaptively adjust the value of $\alpha$. Moreover, to preserve agents' stable trajectories and improve convergence precision, a boundary constraint is derived from the stability conditions of GSA to restrict the value of $\alpha$ in each iteration. The performance of SCAA has been evaluated by comparing with the original GSA and four alpha adjusting algorithms on 13 
conventional functions and 15 complex CEC2015 functions. The experimental results have demonstrated that SCAA has significantly better searching performance than its peers do.

Keywords: Meta-heuristic algorithm, Gravitational Search Algorithm, Adaptive parameter, Stability Conditions, Exploration and exploitation

\section{Introduction}

With the growing complexity in many real-word optimization problems, adaptable and flexible meta-heuristic algorithms are of increasing popularity due to their efficient performances [5,34]. In recent years, a variety of meta-heuristic algorithms have been proposed, including Genetic Algorithm (GA) [3], Particle Swarm Optimization (PSO) [34], Differential Evolution (DE) [45], Artificial Bee Colony (ABC) [19] and Gravitational search algorithm (GSA) [35], etc. Among these algorithms, GSA is one of the latest population-based stochastic algorithm that originates from the Newton's law of gravity and motion [35]. GSA considers every agent as a celestial body attracting each other with a gravitational force that is directly proportional to the product of their masses and inversely proportional to the squared distance between them. Agents search for the optimum by their interactive movements. Since it was developed, GSA has gained popularity due to its several attractive features, such as simple structure, easy implementation and well understanding $[9,18]$. However, there are still some drawbacks in GSA, especially the premature convergence and imbalance of exploration and exploitation $[9,22,55,56]$.

Recently, numerous improvements have been proposed to overcome these drawbacks. One active research trend is to hybridize GSA with other meta-heuristics algorithms, such as DE [25], PSO [4,17,31,32], GA [39,47], ABC [12] and Simulated Annealing (SA) [24]. For example, Li et al. [25] incorporated both the concepts of DE and GSA 
and proposed a hybrid DE-GSA approach, in which agents were updated not only by DE operators but also by GSA mechanisms. Mirjalili et al. introduced the social thinking of PSO into GSA to accelerate convergence in the last iterations and improve the search ability [4,17,32]. In [39,47], GSA was hybridized with GA to escape from local optima when applied to cope with multi-level image thresholding and neural network training issues, respectively.

Another research trend is to introduce new learning strategies into GSA. To tackle the prematurity problem of GSA, Sun et al. [48] presented a locally information topology by taking individual heterogeneity into account and Doraghinejad et al. [7] embedded the Black Hole theory into the original GSA. Sarafrazi et al. [37] defined a new operator named as "disruption" to increase the exploration and exploitation ability of GSA. For overcoming the limitation of lack of historical memory in GSA, the information of agents' best solution obtained so far was introduced in [18]. Xiao et al. [53] modified GSA by introducing the chaotic local search operator to avoid the local optima trapping problem. Besides, Soleimanpour-moghadam et al. [42] proposed a Quantum based GSA for increasing the population diversity. For striking a good balance between exploration and exploitation, Khajezadeh et al [20] developed a modified GSA (IGSA) by introducing a controlled trajectory for velocity update that limited the velocity within a certain interval value.

In addition, there is another strong research trend towards designing new parameter adjusting strategies of GSA to improve its performance. In GSA, the gravitational constant $G^{t}$ determines the convergence speed and the balance of exploration and exploitation. In order to improve the search ability of GSA, several linearly decreasing functions of $G^{t}$ were used in [13,14] to extend the solution search space. Li et al. [23] proposed a piecewise function based GSA (PFGSA) for providing more rational gravitational constant to control the convergence. Vijaya Kumar et al. [52] developed a fuzzy adaptive GSA (FAGSA), where fuzzy rules were used to determine the optimal 
selection of gravitational constant. More specially, by adjusting the attenuation factor alpha $(\alpha), G^{t}$ is correspondingly changed and leads to the alteration of agents' movement directions and steps [2,36]. Thus, the parameter $\alpha$ plays an important role in the searching ability of GSA. However, a constant parameter $\alpha$ was used in the original GSA in the whole evolutionary process, which may severely affect the optimization performance. To address this limitation, a number of alpha adjusting strategies have been proposed. In [43] and [11], a fuzzy strategy was used to adjust the $\alpha$ value on the basis of the iteration number for the sake of promoting the balance of exploration and exploitation and discouraging the premature convergence. In [23] and [56], a hyperbolic function was introduced to replace the fixed value of $\alpha$, which requested $\alpha$ to be changed with iteration to tackle the premature problem. Besides, Saeidi-Khabisi et al. [36] proposed an adaptive alpha determination strategy by using a fuzzy logic controller. In this method, some feedback information including the current iteration value, population diversity, population progress and the $\alpha$ value in the previous iteration were utilized to adjust $\alpha$ dynamically, which aimed at accelerating the convergence rate and preventing prematurity. More comprehensive and detailed overview of the GSA variants can be found in $[33,40]$.

Nevertheless, the aforementioned alpha adjusting methods have mitigated but not solved the premature convergence. One key issue is that most of them adopt the same $\alpha$ value for all agents in each iteration without considering their evolutionary states. Moreover, very limited focus has been put on the stability of GSA, though it actually promotes the convergence speed and precision. After having elaborated investigation of the parameter $\alpha$ and the stability conditions, a new adaptive alpha adjusting strategy, stability constrained adaptive alpha for GSA (SCAA), is introduced in this paper to enhance the performance of GSA. The novel contributions of the proposed SCAA are highlighted in two aspects as follows: 
(1) An adaptive alpha adjusting strategy: In SCAA, the evolutionary state of each agent is first estimated. According to the estimated state, the variation of the agent's position and fitness are used as feedback to adaptively adjust its $\alpha$ value. Consequently, the novel alpha adjusting method can accelerate the convergence speed and alleviate the premature problem.

(2) Stability-based boundary constraint for parameter $\alpha$ : For further improving the convergence speed and precision, a boundary constraint on the basis of stability conditions is presented to restrict the $\alpha$ value in each iteration. Experimental results show that this $\alpha$ boundary constraint ensures the stable convergence.

The remainder of this paper is organized as follows. Section 2 provides some preliminaries of GSA. The detail of the proposed method is discussed in Section 3. In Section 4, experimental results and stability analysis are given to evaluate the proposed algorithm. Finally, some concluding remarks are drawn in Section 5.

\section{Gravitational search algorithm}

GSA is a population-based meta-heuristic algorithm motivated by the laws of gravity and mass interactions [35]. In GSA, every agent $\boldsymbol{X}_{i}=\left[x_{i 1}, \ldots, x_{i d}, \ldots, x_{i D}\right](i=1,2, \ldots, N P)$ attracts each other by a medium called gravitational force in a $D$-dimensional search space. The gravitational force is directly proportional to their masses and inversely proportional to their squared distance [35,36,43]. Accordingly, agents tend to move towards other agents with heavier masses, which are corresponding to good solutions in the search space [31,32]. The mass of the $i$-th agent in the iteration $t, M_{i}^{t}$, is calculated as follows: 


$$
\begin{gathered}
\operatorname{mass}_{i}^{t}=\frac{\text { fit }_{i}^{t}-\text { worst }^{t}}{\text { best }^{t}-\text { worst }^{t}} \\
M_{i}^{t}=\frac{\operatorname{mass}_{i}^{t}}{\sum_{j=1}^{N P} \operatorname{mass}_{j}^{t}}
\end{gathered}
$$

where $f i t_{i}^{t}$ is the fitness value of the $i$-th agent in the iteration $t$. For the minimum problem, best $^{t}=\min _{j \in\{1, \ldots, N P\}} f i t_{j}^{t}$, worst $^{t}=\max _{j \in\{1, \ldots, N P\}} f i t_{j}^{t}$.

During all epochs, the gravitational force exerted on the $i$-th agent from the $j$-th agent at a specific time $t$ is defined by Eq. (3).

$$
F_{i d . j d}^{t}=G^{t} \frac{M_{i}^{t} \times M_{j}^{t}}{\left\|\boldsymbol{X}_{i}^{t}, \boldsymbol{X}_{j}^{t}\right\|_{2}+\varepsilon}\left(x_{i d}^{t}-x_{j d}^{t}\right)
$$

where $M_{j}^{t}$ and $M_{i}^{t}$ are the gravitational mass related to the $i$-th agent and $j$-th agent, respectively. $G^{t}$ is the gravitational constant in the iteration $t,\left\|\boldsymbol{X}_{i}^{t}, \boldsymbol{X}_{j}^{t}\right\|_{2}$ is the Euclidian distance between the $i$-th agent and $j$-th agent, $\varepsilon$ is a small positive constant.

In the $d$-th dimension of the problem space, the total force that acts on the agent $i$ is calculated by:

$$
F_{i d}^{t}=\sum_{j \in K_{\text {best }}, j \neq i}^{N P} \operatorname{rand}_{j} F_{i d, j d}^{t}
$$

where rand $_{j}$ is a random number between the interval [0,1], which is used to provide the random movement step for agents to empower their diverse behaviors. $K_{\text {best }}$ is an archive to store $K$ superior agents (with bigger masses and better fitness values) after fitness sorting in each iteration, whose size is initialized as $N P$ and linearly decreased with time down to one. Thus, by the law of motion, the acceleration of the agent $i$ in the $d$-th dimension in the iteration $t, a_{i d}^{t}$, is calculated by Eq. (5).

$$
a_{i d}^{t}=F_{i d}^{t} / M_{i}^{t}
$$


The gravitational constant $G^{t}$ is defined as follows:

$$
G^{t}=G_{0} \times e^{\left(-\alpha \frac{t}{t_{\max }}\right)}
$$

110 where $\alpha$ is the gravitational constant attenuation factor and $t_{\max }$ is the maximum number of iterations. In the

111 original GSA, $G_{0}$ is set to 100 and $\alpha$ is set to 20. In this way, the gravitational constant $G^{t}$ is initialized to $G_{0}$

112 at the beginning and decreases exponentially into zero with lapse of time.

The velocity and position of the agent $i$ are updated by

$$
\begin{gathered}
v_{i d}^{t+1}=\operatorname{rand}_{i} \times v_{i d}^{t}+a_{i d}^{t} \\
x_{i d}^{t+1}=x_{i d}^{t}+v_{i d}^{t+1}
\end{gathered}
$$

where rand $_{i}$ is a uniform random variable in the interval $[0,1]$ and it can give a randomized characteristic to the search. In this paper, for clearly describing and calculating the stability conditions in Section 3.2, a user-specified inertia weight $w$ is introduced to determine how easily the previous velocity can be changed. Thus, Eq. (7) is rewritten as follows:

$$
v_{i d}^{t+1}=w \times v_{i d}^{t}+a_{i d}^{t}
$$

\section{The proposed SCAA algorithm}


(a), the center $c_{1}^{t}$ is close to the global optimum and the agent $M_{1}^{t}$ has been self-improved in several sequential steps in the current iteration $t$. In this case, the impact of elite masses should be enhanced to strength the movement tendency of $M_{1}^{t}$ towards the center $c_{1}^{t}$ for accelerating convergence. On the contrary, if the elite masses are trapped into local optima, especially in the latter stage when the size of $K_{\text {best }}$ is decreased to a smaller value, their center is more likely far away from the global optimum. As a result, the agent may experience false convergence and its fitness can be worse and worse [32,48] as shown in Fig. 1 (b). In this regard, the impact of elite masses should be weakened to reduce the movement tendency of $M_{1}^{t}$ towards the center $c_{1}^{t}$ for avoiding prematurity.

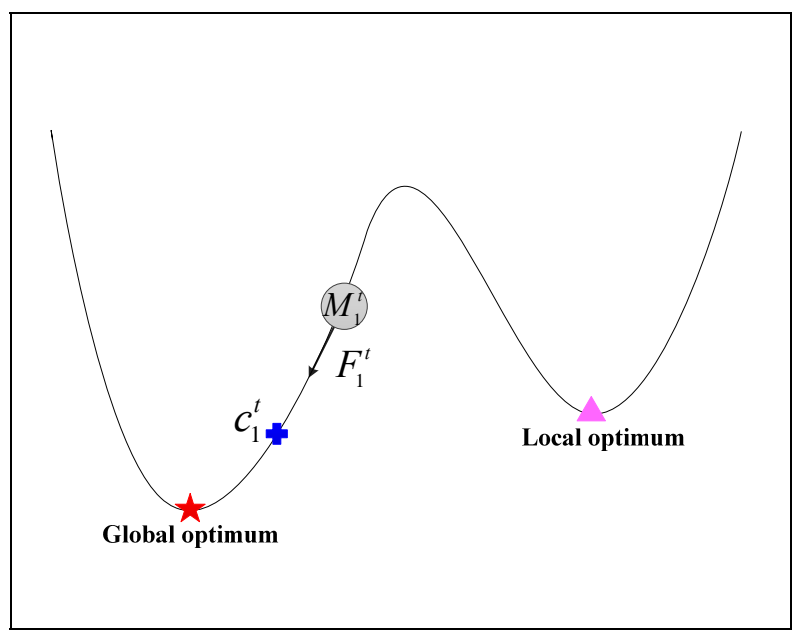

(a) Elite agents' center is close to the global optimum.

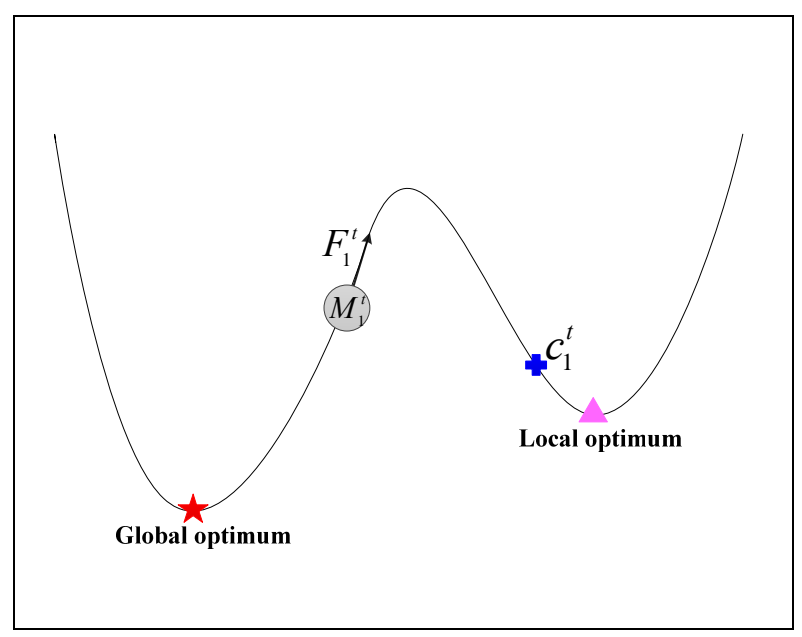

(b) Elite agents' center is far away from the global optimum.

Fig. 1. Schematic diagram of the agent's movement.

As analyzed above, agents may experience different evolutionary states during a course of simulation, which can be defined in two cases: (1) the agent has improved self-solution in several sequential steps and (2) the agent has failed to improve self-solution for several sequential steps. The tendency of an agent moving towards elite masses is supposed to be dynamically changed corresponding to its states for a better convergence. According to Eq. (6), the gravitational constant $G^{t}$ is the modulus of force that controls the impact of elite masses. Moreover, by 
adjusting the attenuation factor alpha $(\alpha), G^{t}$ is also changed accordingly. Specifically, a smaller $\alpha$ value results in a greater $G^{t}$ that promotes the agent to move faster toward the center of $K_{\text {best }}$, while a larger $\alpha$ value leads to a lower $G^{t}$ that prevents the agent from reaching the center [9,32]. Therefore, in this paper, the parameter $\alpha$ is adaptively adjusted according to the agent’s current state.

In order to estimate agents' evolutionary states, two counters, $n s$ and $n f$, are used as the indicators. For a given agent $i$, the $n s_{i}^{t}$ and $n f_{i}^{t}$ are both set to zero initially. Then, as described in Eqs. (10) and (11), if $\boldsymbol{X}_{i}^{t}$ can improve self-solution in the new iteration, $n s_{i}^{t}$ is incremented by 1 and $n f_{i}^{t}$ is set to 0 . Otherwise, the counter $n f_{i}^{t}$ is incremented by 1 whilst $n s_{i}^{t}$ is set to 0 . Obviously, the values of $n s_{i}^{t}$ and $n f_{i}^{t}$ indicate the different evolutionary states of the agent $i$.

$$
\begin{aligned}
n s_{i}^{t} & = \begin{cases}n s_{i}^{t-1}+1, & \text { if } \text { fit }_{i}^{t}<\text { fit }_{i}^{t-1} \\
0, & \text { otherwise }\end{cases} \\
n f_{i}^{t} & = \begin{cases}n f_{i}^{t-1}+1, & \text { if } \text { fit }_{i}^{t} \geq \text { fit }_{i}^{t-1} \\
0, & \text { otherwise }\end{cases}
\end{aligned}
$$

We set a limit value $l p$ to judge whether or not to conduct the adjustment of $\alpha$. For the $i$-th agent in the iteration $t$, if $n s_{i}^{t}$ exceeds $l p, \boldsymbol{X}_{i}^{t}$ is recognized as in the first case [1,49] and its $\alpha$ value should be decreased to enhance the convergence to elite masses. More specifically, a less variation in fitness or position of agent $\boldsymbol{X}_{i}^{t}$ denotes its slow movement. Thus, its $\alpha$ value should be decreased greatly to reach a higher convergence speed. When the position or fitness of agent $\boldsymbol{X}_{i}^{t}$ change greatly, it means the agent moves faster or locates a more promising region, thus the $\alpha$ value needs be slightly decreased to relatively refine its neighboring areas. On the other hand, if $n f_{i}^{t}$ exceeds $l p, \quad \boldsymbol{X}_{i}^{t}$ is regarded as in the second case [1,49]. The parameter $\alpha$ is supposed to be increased to reduce the attraction of elite masses, i.e. its $\alpha$ value changes just in the opposite way as the first case 

feedback to adaptively adjust the parameter $\alpha$ according to the evolutionary state, which is described in Eq. (12).

$$
\alpha_{i}^{t}= \begin{cases}\alpha_{i}^{t-1}-\operatorname{rand} \times \exp \left(-\frac{\left\|\boldsymbol{X}_{i}^{t}, \boldsymbol{X}_{i}^{t-1}\right\|_{2}}{\max _{j \in\{1, \ldots, N\}}\left\|\boldsymbol{X}_{j}^{t}, \boldsymbol{X}_{j}^{t-1}\right\|_{2}+\varepsilon}\right) \times \exp \left(\frac{f i t_{i}^{t}-f i t_{i}^{t-1}}{\max _{j \in\{1, \ldots, N\}}\left(f i t_{j}^{t-1}-f i t_{j}^{t}\right)+\varepsilon}\right), & \text { if } n s_{i}^{t} \geq l p \\ \alpha_{i}^{t-1}+\operatorname{rand} \times\left(1-\exp \left(-\frac{\left\|\boldsymbol{X}_{i}^{t}, \boldsymbol{X}_{i}^{t-1}\right\|_{2}}{\max _{j \in\{1, \ldots, N\}}\left\|\boldsymbol{X}_{j}^{t}, \boldsymbol{X}_{j}^{t-1}\right\|_{2}+\varepsilon}\right)\right) \times\left(1-\exp \left(\frac{f i t_{i}^{t-1}-f i t_{i}^{t}}{\max _{j \in\{1, \ldots, N\}}\left(f i t_{j}^{t}-f i t_{j}^{t-1}\right)+\varepsilon}\right)\right), & \text { if } n f_{i}^{t} \geq l p \\ \alpha_{i}^{t-1}, & \text { otherwise }\end{cases}
$$

where $\alpha_{i}^{t}$ is the alpha value of the $i$-th agent in the $t$-th iteration, rand is a random value in $[0,1]$ that can enhance the diversity of $\alpha_{i}^{t}$ [21]. From Eq. (12), it is obvious that $\alpha_{i}^{t}$ can be dynamically changed in coincidence with its evolutionary state.

\subsection{Stability-based boundary constraint}

In GSA, the stability conditions play an important role in promoting the convergence speed and precision. In this section, after having elaborated investigation of the stability conditions, we present a boundary constraint for parameter $\alpha$ to enhance the stable convergence of GSA.

As proven in [18], for the $i$-th agent in the $t$-th iteration, gravitational constant $G^{t}$ has to satisfy Eq. (13) to ensure the stability of its movement trajectory.

$$
0<G^{t}<\frac{4(1+w) \times\left(R_{i, j}+\varepsilon\right)}{\sum_{j \in B_{i}} M_{p_{j}}^{t}+\sum_{j \in W_{i}} M_{p_{j}}^{t}}
$$


where $w$ satisfies the stability condition: $0 \leq w<1, \quad B_{i}$ is a set of agents which own better fitness than the agent $i$, $W_{i}$ is a set of agents whose fitness values are no better than the agent $i . M_{p_{j}}^{t}$ is the personal best fitness history found so far by the agent $j$, which is calculated as follows:

$$
\begin{gathered}
p_{j d}^{t+1}= \begin{cases}p_{j d}^{t}, & \text { if } \text { fit }_{p}^{t+1}>\text { fit }_{p_{j}}^{t} \\
x_{j d}^{t+1}, & \text { if } \text { fit }_{p}^{t+1} \leq f i t_{p_{j}}^{t}\end{cases} \\
\text { mass }_{j}^{t}=\frac{\text { fit }_{p_{j}}^{t}-\text { worst }^{t}}{\text { best }^{t}-\text { worst }^{t}} \\
M_{p_{j}}^{t}=\frac{\text { mass }_{j}^{t}}{\sum_{k=1}^{N} \text { mass }_{k}^{t}}
\end{gathered}
$$
gravitational constant equation in Eq. (6), we can rewrite Eq. (13) as follows:

$$
\begin{aligned}
& 0<G_{0} \times e^{\left(-\alpha \frac{t}{t_{\max }}\right)}<\frac{4(1+w) \times\left(R_{i, j}+\varepsilon\right)}{\sum_{j \in B_{i}} M_{p_{j}}^{t}+\sum_{j \in W_{i}} M_{p_{j}}^{t}} \\
& \Rightarrow 0<e^{\left(-\alpha \frac{t}{t_{\max }}\right)}<\frac{4(1+w) \times\left(R_{i, j}+\varepsilon\right)}{G_{0} \times\left(\sum_{j \in B_{i}} M_{p_{j}}^{t}+\sum_{j \in W_{i}} M_{p_{j}}^{t}\right)} \\
& \Rightarrow \frac{t_{\max }}{t} \ln \left(\frac{G_{0} \times\left(\sum_{j \in B_{i}} M_{p_{j}}^{t}+\sum_{j \in W_{i}} M_{p_{j}}^{t}\right)}{4(1+w) \times\left(R_{i, j}+\varepsilon\right)}\right)<\alpha<\operatorname{Inf}
\end{aligned}
$$

where $w$ is set to a certain value in the range $[0,1)$. For simplicity, we define $\alpha_{\min }^{t}=\frac{t_{\max }}{t} \ln \left(G_{0} \times\left(\sum_{j \in B_{i}} M_{p_{j}}^{t}+\sum_{j \in W_{i}} M_{p_{j}}^{t}\right) / 4(1+w) \times\left(R_{i, j}+\varepsilon\right)\right)$. Eq. (17) offers the lower boundary that $\alpha$ should be satisfied. Thus, for the agent $i$ in current iteration $t$, if its alpha value is lower than $\alpha_{\min }^{t}$, $\alpha_{i}^{t}$ will be restricted to its boundary:

$$
\alpha_{i}^{t}=\alpha_{\min }^{t}, \quad \text { if } \quad \alpha_{i}^{t}<\alpha_{\text {min }}^{t}
$$


Eq. (18) formulates the lower boundary constraint for parameter $\alpha$. However, there is lack of an upper boundary constraint for $\alpha$. In practice, too large value of $\alpha$ can cause the search to the stagnation and impair the exploration capability. Therefore, it is unreasonable to set the upper boundary value to the infinite great as shown in Eq. (17). To resolve this problem, we set a parameter $\alpha_{\max }$ to control the upper boundary of $\alpha$. Note $\alpha_{\max }$ is fixed to a certain value. In such a way, the boundary constraint equation of $\alpha$, Eq. (17), can now be rewritten as:

$$
\alpha_{\min }^{t} \leq \alpha_{i}^{t} \leq \alpha_{\max }
$$

If $\alpha_{i}^{t}$ is larger than $\alpha_{\max }$, it will be conditioned to its upper boundary as follows:

$$
\alpha_{i}^{t}=\alpha_{\max }, \quad \text { if } \alpha_{i}^{t}>\alpha_{\max }
$$

The sensibility tests on $\alpha_{\max }$ in Section 4.4 verify its effectiveness on the performance of GSA. In a word, the parameter $\alpha_{i}^{t}$ should satisfy the boundary conditions as show in Eq. (19). If the $\alpha_{i}^{t}$ value exceeds its boundary, $\alpha_{i}^{t}$ will be forced to gather on its corresponding boundary as described in Eqs. (18) and (20). Thus, the stability-based boundary constraint can ensure the stable convergence of the swarm.

Based on the above introduction of the SCAA algorithm, its complete pseudo-code is summarized in Algorithm 1 as follows.

Algorithm 1 Pseudo-code of SCAA

1 Generate uniformly distributed population randomly and initialize the velocity associated with each agent;

2 Calculate the fitness value of each agent and generate the $K_{\text {best }}$ agents of the population;

3 Set $F E s=N P, t=1, n s=0, n f=0$; 


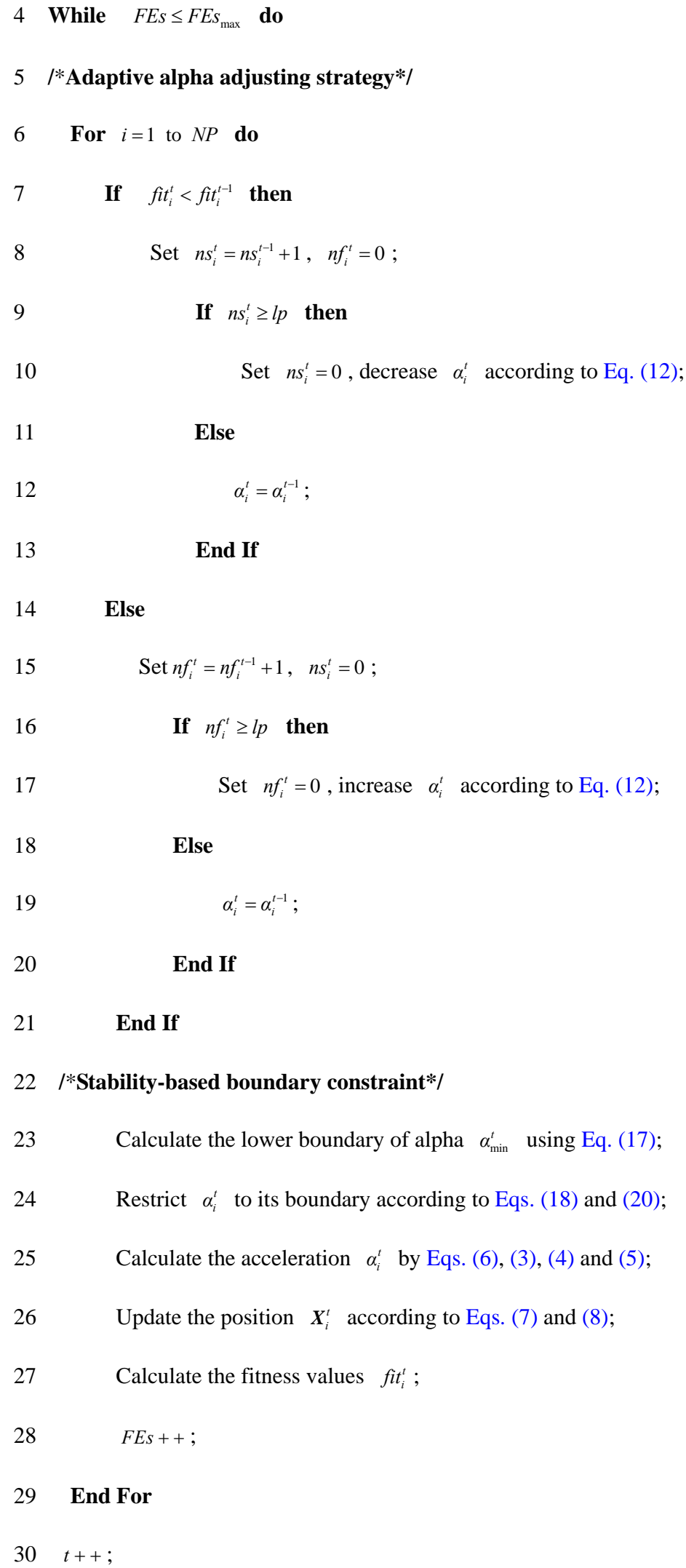

End If

\section{$21 \quad$ End If}

/*Stability-based boundary constraint*/

23

Calculate the lower boundary of alpha $\alpha_{\min }^{t}$ using Eq. (17);

24 Restrict $\alpha_{i}^{t}$ to its boundary according to Eqs. (18) and (20);

$25 \quad$ Calculate the acceleration $\alpha_{i}^{t}$ by Eqs. (6), (3), (4) and (5);

$26 \quad$ Update the position $\boldsymbol{X}_{i}^{t}$ according to Eqs. (7) and (8);

$27 \quad$ Calculate the fitness values $\mathrm{fit}_{i}^{t}$;

$28 \quad F E s++$;

29 End For

$30 t++$;

\section{End While}

32 Output the best solution achieved so far. 
In this section, the search behaviors of SCAA are investigated so as to validate the effectiveness of the proposed

alpha adjusting strategy. We herein take a time-varying 30-D Sphere function as an example.

$$
f(x-r)=\sum_{j=1}^{D}\left(x_{j}-r\right)^{2}, \quad x_{j} \in[-100,100]
$$

where $r$ is initialized to -10 and shifted to 10 in the $200^{\text {th }}$ iteration with the total number of iterations set as 2000 .

That is, the theoretical minimum of $f$ shifts from $(-10)^{D}$ to $(10)^{D}$ during the evolutionary process. SCAA and GSA are employed with the same initialized population, which include 50 agents to solve this minimization problem. For a better observation, only the first agent's alpha value $\alpha_{1}$ and its first dimension trajectory $x_{11}$ are recorded in Fig. 2 (a) and Fig. 2 (b), respectively. The convergence curve of SCAA and GSA are depicted in Fig. 2 (c).

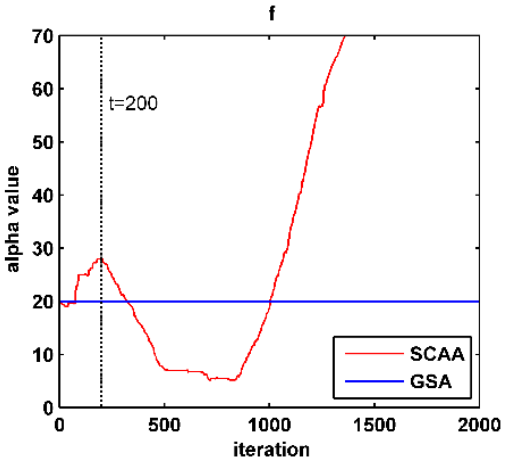

(a) The curve of $\alpha_{1}$ values.

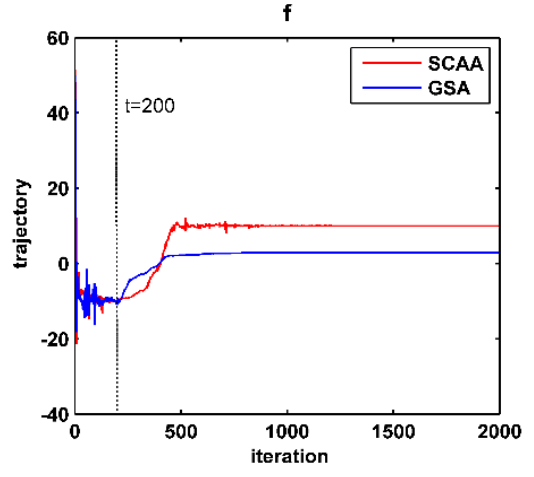

(b) The trajectory curve of agent $x_{11}$.

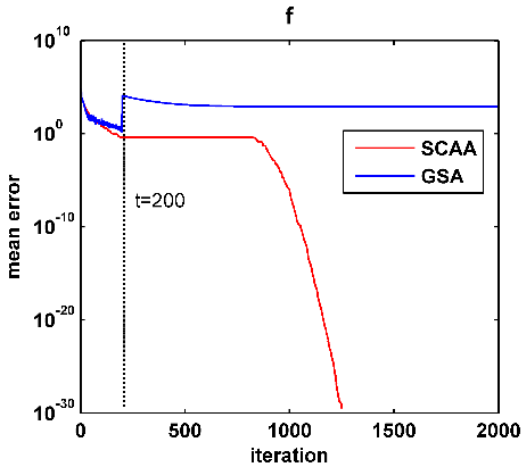

(c) The convergence curve.

Fig. 2. Search behaviors of SCAA on 30- $D$ Sphere function.

During the evolutionary process, the center of elite masses is dynamically changed with agents' movements. In the early iterations, the elite agents may experience poor areas, hence the center is more likely located at the local 
optima. In this case, as shown in Fig. 2 (a), the parameter $\alpha_{1}$ is continuously increased to a higher value before the $200^{\text {th }}$ iteration to weaken the impact of elite masses. This adjustment prevents the agent from being trapped into local optima as revealed in Fig. 2 (b). The $x_{11}$ gradually reaches the global minimum (-10). Thereafter, in the $200^{\text {th }}$ iteration, the value of $r$ is shifted to 10 . At this moment, those agents that are closer to the new global minimum would be the elite masses in $K_{\text {best }}$. To this end, the impact of elite masses should be enhanced to accelerate the convergence. From the Fig. 2 (b) and Fig. 2 (c), it can be seen that parameter $\alpha_{1}$ is rapidly decreased into a smaller value whilst the trajectory of $x_{11}$ deviates from -10 and fast reaches the new global optimum. In the latter iterations, when agents cluster together and converge to the globally optimal area, less improvement of agents' fitness are obtained. Therefore, parameter $\alpha_{1}$ is rapidly increased under the stability-based boundary constraint to improve the convergence precision. As depicted in Fig. 2 (c), SCAA finally achieves the globally optimal results. With regard to the original GSA, even though the swarm can converge to the global optimum in the early iterations, the agents are trapped into local optima after the global minimum is changed. This behavior may mainly result from the usage of a constant $\alpha$, which lacks the dynamic momentum in coincidence with the changed search environment. Conversely, in SCAA, the $\alpha$ value of each agent is adaptively adjusted according to its evolutionary state, which motivates the agent to detect the promising direction and avoid the premature convergence.

\section{Experimental verification and comparison}

\subsection{Experimental setup}


To fully evaluate the performance of SCAA, a thorough comparison with the original GSA [35] and four recent GSA variants with well-established alpha adjusting strategies, including MGSA- $\alpha$ [23], FS $\alpha$ (Increase) [43], FS $\alpha$ (Decrement) [43] and FuzzyGSA [36], is tested in this study. Note that two alpha adjusting methods in [43], FS $\alpha$ (Increase) and FS $\alpha$ (Decrement), are both introduced for investigating the effect of different alpha change trends on the searching performance and the stability of GSA. The tested functions include 28 scalable benchmark functions, where F1-F13 are conventional problems in [54] and F14-F28 are derived from the CEC2015 functions [26]. Detailed description of these benchmark functions can be found in [26,54]. In this paper, the evaluations are performed under 30 dimensions (30-D) and the accuracy level $\delta$ is set to 0.001 for all benchmark functions.

During the experiments, the parameter configurations of all involved algorithms are set according to their recommended settings [48]. Note that for the fair comparison, MGSA- $\alpha$ only uses the $\alpha$ adjusting strategy and abandons the mutation operator in MGSA. As for SCAA, the initial value of parameter alpha $\alpha_{0}$ is set to 20 for all agents on the basis of the recommendations in [35]. The inertia weight is set to $w=1-1 / t_{\max }$ according to the range in stability conditions as suggested in [18]. The limit value $l p$ and $\alpha_{\max }$ are respectively set to 2 and 70 according to the sensitive analysis conducted in Section 4.5. The detailed parameter settings of all involved algorithms are summarized in Table 1.

In the experiments, common parameters are the total number of trials, maximum number of function evaluations $\left(F E s_{\max }\right)$ and the population size $(N P)$. All algorithms were independently run 51 times to reduce random discrepancy [26]. The $F E s_{\max }$ for terminating the algorithms is specified as $10000 \times D$ for each function as suggested in [26]. The population size $N P$ for solving the $30-D$ problems is set to 50 based on the recommendation in $[35,36,43]$. Moreover, since all involved algorithms have the same fitness evaluations $F E s=N P$ in each iteration, the maximum 
number of iterations $t_{\max }$ is set to $t_{\max }=F E s_{\max } / N P$ in this paper. All the algorithms are implemented using Matlab

\section{Table 1}

Parameter settings of the involved GSA variants.

\begin{tabular}{lcc}
\hline Algorithms & Year & Parameter settings \\
\hline GSA [35] & 2009 & $G_{0}=100, \quad \alpha=20$ \\
FuzzyGSA [36] & 2012 & $E D \in[0,1], \quad C M \in[0,1], \quad t \in\left[0, t_{\max }\right], \quad \alpha \in[29,31]$ \\
FS $\alpha$ (Increase) [43] & 2013 & $t \in[0,100 \%] \times t_{\text {max }}, \quad \alpha \in[0,150]$ \\
FS $\alpha$ (Decrement) [43] & 2013 & $t \in[0,100 \%] \times t_{\max }, \quad \alpha \in[0,150]$ \\
MGSA- $\alpha$ [22] & 2016 & $G_{0}=100, \quad \gamma=0.2, \quad \eta=10, \quad \lambda=25$ \\
SCAA & - & $G_{0}=100, \quad \alpha_{0}=20, \quad w=1-1 / t_{\max }, \quad l p=2, \quad \alpha_{\max }=70$ \\
\hline
\end{tabular}

\subsection{Performance metrics}

In this study, the searching accuracy, searching reliability and searching efficiency of different algorithms are evaluated in terms of the mean error (Mean), success rate $(S R)$, success performance $(S P)$ and execution time (runtime in seconds), respectively [46]. Mean is the average error between the best output results and the global optimum of the optimization problem [46]. SR represents the percentage of successful runs where the algorithm achieves good solutions under the predefined accuracy level $\delta$ [46]. SP denotes the number of $F E s$ required by an algorithm to achieve the acceptable solutions under $\delta$ [46]. The performance metric runtime is the execution time of an algorithm to obtain the acceptable solutions [27]. Meanwhile, we record the standard deviation $(S D)$ of the optimization error and rank the algorithms from the smallest Mean to the highest. The average ranks and the overall ranks obtained by algorithms are also recorded. 
For the rigorous comparison between SCAA and its competitors, a non-parametric statistical test is used in this study. Unlike parametric tests, non-parametric tests are employed to analyze the performance of stochastic algorithms based on computational intelligence despite the assumptions of data types used are violated [10]. Specifically, the Wilcoxon signed ranks test [6] with a confidence level of $5 \%$ is utilized to perform pairwise comparison between SCAA and its peers in this paper.

\subsection{Comparison with other alpha adjusting GSA variants}

Following the experimental setup and parameter settings in Section 4.1, comprehensive experiments are employed to evaluate the overall searching behaviors of GSA, MGSA- $\alpha$, FuzzyGSA, FS $\alpha$ (Increase), FS $\alpha$ (Decrement) and SCAA. For performance assessment, six metrics are used which include the Mean, $S D$ and Wilcoxon signed ranks test (p-value, $h$-value, $z$-value and signedrank), as summarized in Table 2 and Table 3, along with $S R, S P$ and runtime as reported in Table 4. Besides, we rank the six competing algorithms according to their Mean values. In Table 2 and Table 3, the symbol ' $h$ ' describes the non-parameter test results, where '+' means SCAA is significantly better than the compared algorithms, '-' indicates it significantly performs worse and '=' stands for comparable performance between the algorithms. Moreover, we summarize the results of Wilcoxon test results at the bottom of Table 2 and Table 3, respectively. The best result in each row is highlighted in bold in the Tables 2-4. Note that Fig. 3 depicts some convergence curves of the competing algorithms.

\section{Table 2}

Optimization errors among six algorithms on 13 conventional problems at 30-D. 


\begin{tabular}{|c|c|c|c|c|c|c|c|}
\hline & metrics & GSA & MGSA- $\alpha$ & FuzzyGSA & FS $\alpha$ (Increase) & FS $\alpha$ (Decrement) & SCAA \\
\hline \multirow{5}{*}{ F1 } & Mean (Mean_rank) & 1.188E-17(6) & 4.481E-34(3) & 7.291E-27(5) & 1.322E-38(2) & $6.514 \mathrm{E}-27(4)$ & $9.162 E-58(1)$ \\
\hline & $S D$ & 3.398E-18 & 7.093E-34 & $2.081 \mathrm{E}-27$ & $4.230 \mathrm{E}-38$ & 7.117E-28 & $2.283 E-57$ \\
\hline & & & & & & & \\
\hline & p-value (h-value) & 5.145E-10 (+) & 5.145E-10 (+) & 5.145E-10 (+) & 5.145E-10 (+) & $5.145 \mathrm{E}-10(+)$ & \\
\hline & z-value (signedrank) & $-6.2146(0)$ & $-6.2146(0)$ & $-6.2146(0)$ & $-6.2146(0)$ & $-6.2146(0)$ & \\
\hline \multirow{4}{*}{$\mathrm{F} 2$} & Mean (Mean_rank) & 1.727E-08(6) & 3.038E-16(3) & 4.257E-13(5) & $1.247 \mathrm{E}-18(2)$ & 3.855E-13(4) & 4.558E-20(1) \\
\hline & $S D$ & 2.829E-09 & $2.295 \mathrm{E}-16$ & $5.629 \mathrm{E}-14$ & $1.094 \mathrm{E}-18$ & $1.52 \mathrm{E}-15$ & $9.218 E-20$ \\
\hline & n-value (h-value) & $5.145 \mathrm{E}-10(+)$ & $5.145 \mathrm{E}-10(+)$ & $5.145 \mathrm{E}-10(+)$ & $9662 \mathrm{E}-09(+)$ & $5.145 \mathrm{E}-10(+)$ & \\
\hline & z-value (signedrank) & $-6.2146(0)$ & $-6.2146(0)$ & $-6.2146(0)$ & $-5.7366(51)$ & $-6.2146(0)$ & \\
\hline \multirow{5}{*}{ F3 } & Mean (Mean_rank) & $1.500 \mathrm{E}-02(2)$ & $2.264 \mathrm{E}+00(3)$ & $1.272 \mathrm{E}+01(4)$ & $5.556 \mathrm{E}+01(5)$ & $1.749 \mathrm{E}+02(6)$ & $7.700 \mathrm{E}-03(1)$ \\
\hline & $S D$ & 3.010E-02 & $2.207 \mathrm{E}+00$ & $7.251 \mathrm{E}+00$ & $2.557 \mathrm{E}+01$ & $6.488 \mathrm{E}+01$ & $5.600 \mathrm{E}-03$ \\
\hline & & & & & & & \\
\hline & p-value (h-value) & 1.197E-01 (=) & 5.145E-10 (+) & 5.145E-10 (+) & 5.145E-10 (+) & $5.145 \mathrm{E}-10(+)$ & \\
\hline & z-value (signedrank) & -1.5560 (497) & $-6.2146(0)$ & $-6.2146(0)$ & $-6.2146(0)$ & $-6.2146(0)$ & \\
\hline \multirow{5}{*}{$\mathrm{F} 4$} & Mean (Mean_rank) & 1.823E-09(6) & 5.247E-16(2) & $5.486 \mathrm{E}-14(5)$ & 1.329E-18(1) & 3.385E-14(3) & $5.041 \mathrm{E}-14(4)$ \\
\hline & $S D$ & $2.171 \mathrm{E}-10$ & $2.276 \mathrm{E}-16$ & $9.236 \mathrm{E}-15$ & 6.262E-19 & 2.305E-15 & $2.008 \mathrm{E}-13$ \\
\hline & & & & & & & \\
\hline & p-value (h-value) & 5.145E-10 (+) & $9.104 \mathrm{E}-01(=)$ & 1.309E-05 (+) & 1.768E-09 (-) & 1.309E-05 (-) & \\
\hline & z-value (signedrank) & $-6.2146(0)$ & $0.1125(675)$ & $-4.3587(198)$ & 6.0178 (1305) & $-4.3587(198)$ & \\
\hline \multirow{5}{*}{ F5 } & Mean (Mean_rank) & $1.918 \mathrm{E}+01(2)$ & $2.214 \mathrm{E}+01(3)$ & $2.387 E+01(4)$ & $2.901 E+01(5)$ & $3.223 E+01(6)$ & $1.321 E+01(1)$ \\
\hline & $S D$ & 2.264E-01 & 1.769E-01 & $1.743 \mathrm{E}-01$ & $2.290 \mathrm{E}+01$ & $3.235 \mathrm{E}+01$ & 4.512E-01 \\
\hline & & & & & & & \\
\hline & p-value (h-value) & 5.145E-10 (+) & 5.145E-10 (+) & $5.145 \mathrm{E}-10(+)$ & $5.145 \mathrm{E}-10(+)$ & $5.145 \mathrm{E}-10(+)$ & \\
\hline & z-value (signedrank) & $-6.2146(0)$ & $-6.2146(0)$ & $-6.2146(0)$ & $-6.2146(0)$ & $-6.2146(0)$ & \\
\hline \multirow{5}{*}{ F6 } & Mean (Mean_rank) & $0.00 E+00(1)$ & $0.00 \mathrm{E}+00(1)$ & $0.00 \mathrm{E}+00(1)$ & $0.00 E+00(1)$ & $0.00 E+00(1)$ & $0.00 \mathrm{E}+00(1)$ \\
\hline & $S D$ & $0.00 E+00$ & $0.00 E+00$ & $0.00 E+00$ & $0.00 E+00$ & $0.00 E+00$ & $0.00 E+00$ \\
\hline & & & & & & & \\
\hline & p-value (h-value) & $1.000 \mathrm{E}+00(=)$ & $1.000 \mathrm{E}+00(=)$ & $1.000 \mathrm{E}+00(=)$ & $1.000 \mathrm{E}+00(=)$ & $1.000 \mathrm{E}+00(=)$ & \\
\hline & z-value (signedrank) & $-(0)$ & $-(0)$ & $-(0)$ & $-(0)$ & $-(0)$ & \\
\hline \multirow{5}{*}{ F7 } & Mean (Mean_rank) & $9.24 \mathrm{E}-02(6)$ & 8.18E-02(5) & $1.070 \mathrm{E}-02(2)$ & 1.240E-02(3) & $1.460 \mathrm{E}-02(4)$ & $1.050 \mathrm{E}-02(1)$ \\
\hline & $S D$ & $3.560 \mathrm{E}-02$ & $2.890 \mathrm{E}-02$ & $3.200 \mathrm{E}-03$ & $5.000 \mathrm{E}-03$ & $5.200 \mathrm{E}-03$ & $6.200 \mathrm{E}-03$ \\
\hline & & & & & & & \\
\hline & p-value (h-value) & 5.145E-10 (+) & 5.145E-10 (+) & 8.293E-01 (=) & 1.153E-01 (=) & 2.759E-04 (+) & \\
\hline & z-value (signedrank) & $-6.2146(0)$ & $-6.2146(0)$ & $-0.2156(640)$ & $-1.5747(495)$ & $-3.6369(275)$ & \\
\hline
\end{tabular}




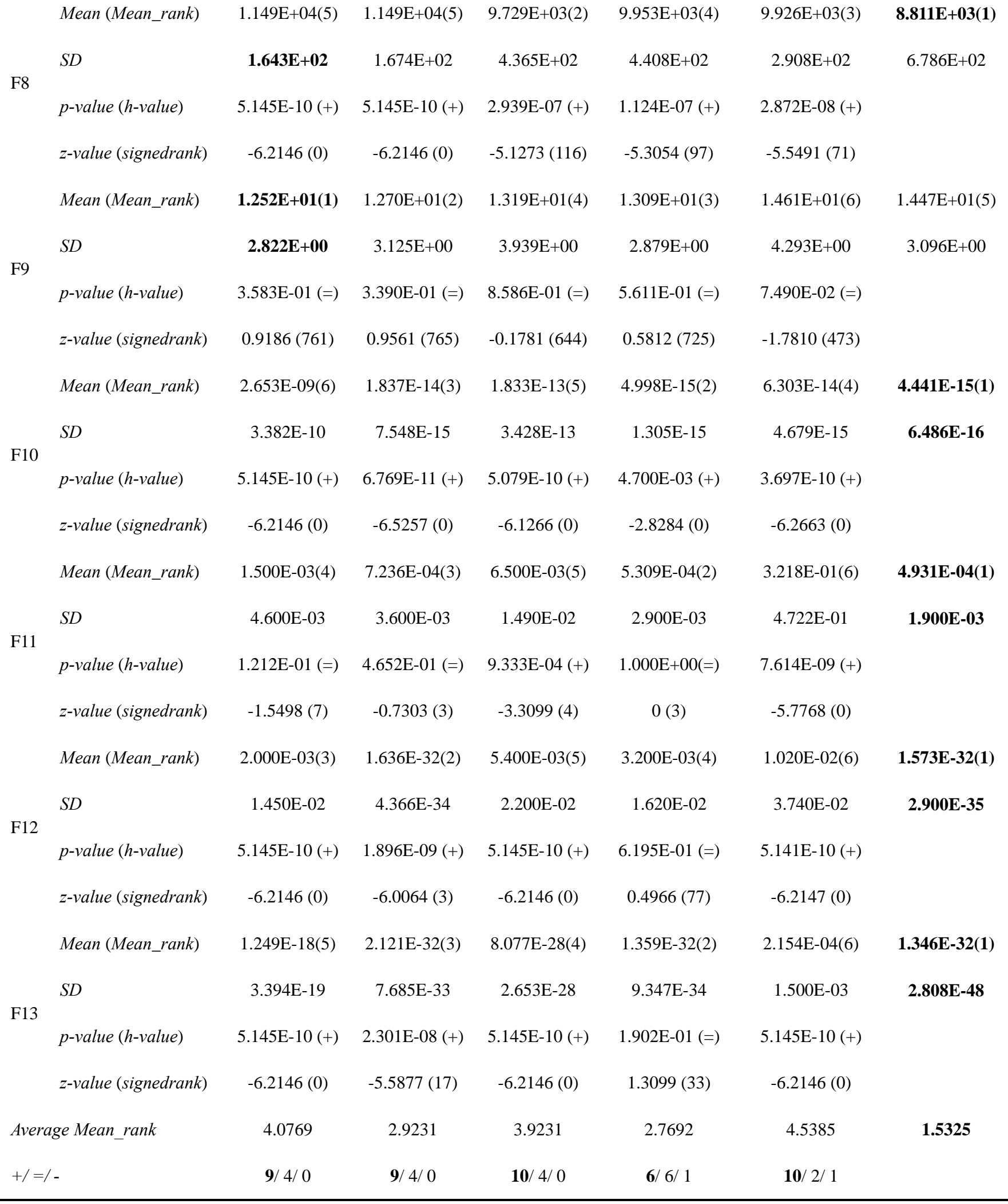

\section{Table 3}

Optimization errors among six algorithms on 15 CEC2015 functions at 30-D. 


\begin{tabular}{|c|c|c|c|c|c|c|c|}
\hline & metrics & GSA & MGSA- $\alpha$ & FuzzyGSA & FS $\alpha$ (Increase) & FS $\alpha$ (Decrement) & SCAA \\
\hline \multirow{5}{*}{ F14 } & Mean (Mean_rank) & $8.737 \mathrm{E}+05(2)$ & $1.012 \mathrm{E}+06(3)$ & $2.707 \mathrm{E}+06(5)$ & $2.046 \mathrm{E}+06(4)$ & $1.428 \mathrm{E}+07(6)$ & $4.093 E+05(1)$ \\
\hline & $S D$ & $4.497 \mathrm{E}+05$ & $3.907 E+05$ & $5.063 \mathrm{E}+06$ & $1.297 \mathrm{E}+06$ & $8.813 E+06$ & $2.405 E+05$ \\
\hline & & & & & & & \\
\hline & p-value (h-value) & 1.273E-08 (+) & $9.141 \mathrm{E}-09(+)$ & 4.673E-09 (+) & 5.145E-10 (+) & $5.145 \mathrm{E}-10(+)$ & \\
\hline & z-value (signedrank) & $-5.6897(56)$ & $-5.7459(50)$ & $-5.5884(38)$ & $-6.2146(0)$ & $-6.2146(0)$ & \\
\hline \multirow{5}{*}{ F15 } & Mean (Mean_rank) & $4.729 \mathrm{E}+02(2)$ & $7.445 \mathrm{E}+02(5)$ & $7.169 \mathrm{E}+02(4)$ & $7.634 \mathrm{E}+02(6)$ & $4.701 E+02(1)$ & $5.328 \mathrm{E}+02(3)$ \\
\hline & $S D$ & $4.771 E+02$ & $9.367 \mathrm{E}+02$ & $9.480 \mathrm{E}+02$ & $1.117 \mathrm{E}+03$ & $6.262 \mathrm{E}+02$ & $8.585 E+02$ \\
\hline & & & & & & & \\
\hline & p-value (h-value) & 5.486E-01 (=) & $3.250 \mathrm{E}-01(=)$ & 2.489E-01 (=) & $2.528 \mathrm{E}-01(=)$ & 7.858E-01 (=) & \\
\hline & z-value (signedrank) & -0.5999 (599) & $-0.9842(558)$ & $-1.1529(540)$ & $-1.1436(541)$ & $-0.2718(634)$ & \\
\hline \multirow{5}{*}{ F16 } & Mean (Mean_rank) & $2.00 \mathrm{E}+01(1)$ & $2.00 \mathrm{E}+01(1)$ & $2.00 E+01(1)$ & $2.00 \mathrm{E}+01(1)$ & $2.00 \mathrm{E}+01(1)$ & $2.094 \mathrm{E}+01(2)$ \\
\hline & $S D$ & $9.746 \mathrm{E}-05$ & $6.594 \mathrm{E}-05$ & 8.113E-05 & 1.109E-04 & 6.150E-05 & $5.840 \mathrm{E}-02$ \\
\hline & & & & & & & \\
\hline & p-value (h-value) & 5.145E-10 (-) & 5.145E-10 (-) & 5.145E-10 (-) & $5.145 \mathrm{E}-10(-)$ & 5.145E-10 (-) & \\
\hline & z-value (signedrank) & $6.2146(1326)$ & 6.2146 (1326) & $6.2146(1326)$ & $6.2146(1326)$ & $6.2146(1326)$ & \\
\hline \multirow{5}{*}{ F17 } & Mean (Mean_rank) & $2.073 \mathrm{E}+02(2)$ & $1.963 E+02(1)$ & $2.194 \mathrm{E}+02(5)$ & $2.084 \mathrm{E}+02(3)$ & $2.363 \mathrm{E}+02(6)$ & $2.173 E+02(4)$ \\
\hline & $S D$ & $2.201 \mathrm{E}+01$ & $2.811 \mathrm{E}+01$ & $1.924 \mathrm{E}+01$ & $2.023 \mathrm{E}+01$ & $2.261 \mathrm{E}+01$ & $2.223 \mathrm{E}+01$ \\
\hline & & & & & & & \\
\hline & p-value (h-value) & 2.830E-02 (+) & 5.617E-04 (-) & 4.148E-01 (=) & 4.900E-03 (+) & $1.215 \mathrm{E}-04(+)$ & \\
\hline & z-value (signedrank) & $-2.1934(429)$ & $-3.4494(295)$ & $-0.8155(576)$ & $-2.8120(363)$ & $-3.8431(253)$ & \\
\hline \multirow{5}{*}{ F18 } & Mean (Mean_rank) & $3.818 \mathrm{E}+03(4)$ & $3.625 E+03(1)$ & $3.981 \mathrm{E}+03(5)$ & $3.773 \mathrm{E}+03(2)$ & $4.156 \mathrm{E}+03(6)$ & $3.810 \mathrm{E}+03(3)$ \\
\hline & $S D$ & $4.518 \mathrm{E}+02$ & $4.456 \mathrm{E}+02$ & $4.533 E+02$ & $5.325 \mathrm{E}+02$ & $4.548 \mathrm{E}+02$ & $4.548 \mathrm{E}+02$ \\
\hline & & & & & & & \\
\hline & p-value (h-value) & 7.930E-01 (=) & $2.010 \mathrm{E}-02(-)$ & $7.190 \mathrm{E}-02(=)$ & $9.030 \mathrm{E}-01(=)$ & 7.916E-04 (+) & \\
\hline & z-value (signedrank) & $-0.2625(635)$ & $-2.3246(415)$ & $-1.7997(471)$ & $-0.1219(650)$ & $-3.3557(305)$ & \\
\hline \multirow{5}{*}{ F19 } & Mean (Mean_rank) & $1.302 \mathrm{E}+05(2)$ & $3.553 \mathrm{E}+05(3)$ & $6.856 \mathrm{E}+05(4)$ & $9.472 \mathrm{E}+05(5)$ & $1.704 \mathrm{E}+06(6)$ & $5.587 E+04(1)$ \\
\hline & $S D$ & $6.396 \mathrm{E}+04$ & $1.725 \mathrm{E}+05$ & $2.962 \mathrm{E}+05$ & $3.593 E+05$ & $6.204 \mathrm{E}+05$ & $2.566 E+04$ \\
\hline & & & & & & & \\
\hline & p-value (h-value) & $1.420 \mathrm{E}-08(+)$ & 5.145E-10 (+) & 5.145E-10 (+) & 5.145E-10 (+) & 5.145E-10 (+) & \\
\hline & z-value (signedrank) & $-5.6709(58)$ & $-6.2146(0)$ & $-6.2146(0)$ & $-6.2146(0)$ & $-6.2146(0)$ & \\
\hline \multirow{5}{*}{ F20 } & Mean (Mean_rank) & $1.324 \mathrm{E}+01(2)$ & $1.524 \mathrm{E}+01(3)$ & $2.236 \mathrm{E}+01(4)$ & $2.441 \mathrm{E}+01(5)$ & $6.397 \mathrm{E}+01(6)$ & $9.779 E+00(1)$ \\
\hline & $S D$ & $4.061 \mathrm{E}+00$ & $9.643 \mathrm{E}+00$ & $1.949 \mathrm{E}+01$ & $2.058 \mathrm{E}+01$ & $2.388 \mathrm{E}+01$ & $3.133 E+00$ \\
\hline & & & & & & & \\
\hline & p-value (h-value) & $6.522 \mathrm{E}-05(+)$ & 1.669E-06 (+) & $6.142 \mathrm{E}-07(+)$ & 3.371E-08 (+) & 5.145E-10 (+) & \\
\hline & z-value (signedrank) & -3.9931 (237) & $-4.7898(152)$ & $-4.9867(131)$ & $-5.5210(74)$ & $-6.2146(0)$ & \\
\hline
\end{tabular}




\begin{tabular}{|c|c|c|c|c|c|c|c|}
\hline & Mean (Mean_rank) & $2.356 \mathrm{E}+04(2)$ & $2.388 \mathrm{E}+04(3)$ & $3.050 \mathrm{E}+04(4)$ & $5.557 \mathrm{E}+04(5)$ & $1.007 \mathrm{E}+05(6)$ & $2.154 E+04(1)$ \\
\hline & $S D$ & $1.079 E+04$ & $7.509 E+03$ & $1.152 \mathrm{E}+04$ & $3.344 \mathrm{E}+04$ & $1.141 \mathrm{E}+05$ & $8.329 E+03$ \\
\hline F21 & & & & & & & \\
\hline & p-value (h-value) & 2.728E-01 (=) & 6.760E-02 (=) & $1.201 \mathrm{E}-06(+)$ & 5.797E-10 (+) & 5.145E-10 (+) & \\
\hline & z-value (signedrank) & $-1.0967(546)$ & $-1.8278(468)$ & $-4.8555(145)$ & $-6.1959(2)$ & $-6.2146(0)$ & \\
\hline & Mean (Mean_rank) & $1.511 \mathrm{E}+02(4)$ & $1.265 \mathrm{E}+02(2)$ & $1.513 \mathrm{E}+02(5)$ & $1.262 \mathrm{E}+02(1)$ & $2.025 \mathrm{E}+02(6)$ & $1.358 \mathrm{E}+02(3)$ \\
\hline & $S D$ & $1.218 \mathrm{E}+02$ & $8.221 \mathrm{E}+01$ & $1.218 \mathrm{E}+02$ & $7.943 E+01$ & $1.627 \mathrm{E}+02$ & $1.016 \mathrm{E}+02$ \\
\hline F22 & & & & & & & \\
\hline & p-value (h-value) & $1.700 \mathrm{E}-03(+)$ & 6.080E-02 (=) & 7.446E-06 (+) & 1.469E-04 (-) & $1.425 \mathrm{E}-05(+)$ & \\
\hline & z-value (signedrank) & -3.1307 (329) & $-1.8747(463)$ & $-4.4805(185)$ & $-3.7963(258)$ & $-4.3399(200)$ & \\
\hline & Mean (Mean_rank) & $4.299 \mathrm{E}+05(2)$ & $6.936 \mathrm{E}+05(3)$ & $9.961 \mathrm{E}+0545)$ & $1.280 \mathrm{E}+06(5)$ & $2.485 \mathrm{E}+06(6)$ & $1.921 E+05(1)$ \\
\hline & $S D$ & $1.961 \mathrm{E}+05$ & $2.310 \mathrm{E}+05$ & $3.994 \mathrm{E}+05$ & $6.108 \mathrm{E}+05$ & $1.004 \mathrm{E}+06$ & $5.998 E+04$ \\
\hline F23 & & & & & & & \\
\hline & p-value (h-value) & $9.869 \mathrm{E}-10(+)$ & 5.145E-10 (+) & $5.145 \mathrm{E}-10(+)$ & $5.462 \mathrm{E}-10(-)$ & 5.145E-10 (+) & \\
\hline & z-value (signedrank) & $-6.115(11)$ & $-6.2146(0)$ & $-6.2146(0)$ & $-6.2052(1)$ & $-6.2146(0)$ & \\
\hline & Mean (Mean_rank) & $3.244 \mathrm{E}+02(2)$ & $3.343 \mathrm{E}+02(3)$ & $3.480 \mathrm{E}+02(5)$ & $3.473 E+02(4)$ & $3.849 \mathrm{E}+02(6)$ & $3.226 E+02(1)$ \\
\hline & $S D$ & $9.771 \mathrm{E}+01$ & $1.121 \mathrm{E}+02$ & $1.557 \mathrm{E}+02$ & $1.422 \mathrm{E}+02$ & $2.021 \mathrm{E}+02$ & $9.132 E+01$ \\
\hline F24 & & & & & & & \\
\hline & p-value (h-value) & $6.080 \mathrm{E}-02(=)$ & 6.675E-04 (+) & $1.272 \mathrm{E}-08(+)$ & 8.648E-09 (+) & 8.648E-09 (+) & \\
\hline & z-value (signedrank) & $-1.8747(463)$ & $-3.4026(300)$ & $-5.6897(56)$ & $-5.7553(49)$ & $-5.7553(49)$ & \\
\hline & Mean (Mean_rank) & $1.040 \mathrm{E}+02(3)$ & $1.036 \mathrm{E}+02(2)$ & $1.053 \mathrm{E}+02(5)$ & $1.047 \mathrm{E}+02(4)$ & $1.491 \mathrm{E}+02(6)$ & $1.034 E+02(1)$ \\
\hline & $S D$ & 8.472E-01 & $8.215 \mathrm{E}-01$ & $1.104 \mathrm{E}+00$ & $9.304 \mathrm{E}-01$ & $2.771 \mathrm{E}+01$ & 7.031E-01 \\
\hline F25 & & & & & & & \\
\hline & p-value (h-value) & 2.473E-04 (+) & 2.302E-01 (=) & 1.873E-09 (+) & $2.070 \mathrm{E}-07(+)$ & $5.145 \mathrm{E}-10(+)$ & \\
\hline & z-value (signedrank) & $-3.6650(272)$ & $-1.1998(535)$ & $-6.0084(22)$ & $-5.1929(109)$ & $-6.2146(0)$ & \\
\hline & Mean (Mean_rank) & $5.527 \mathrm{E}+03(6)$ & $4.759 \mathrm{E}+03(5)$ & $1.673 \mathrm{E}+03(3)$ & $1.602 \mathrm{E}+03(2)$ & $2.100 \mathrm{E}+03(4)$ & $1.550 E+03(1)$ \\
\hline & $S D$ & $3.988 \mathrm{E}+03$ & $3.987 \mathrm{E}+03$ & $1.083 E+03$ & $1.571 \mathrm{E}+03$ & $1.121 \mathrm{E}+03$ & $1.296 \mathrm{E}+03$ \\
\hline F26 & & & & & & & \\
\hline & p-value (h-value) & 2.905E-06 (+) & $1.425 \mathrm{E}-05(+)$ & $6.528 \mathrm{E}-01(=)$ & $6.528 \mathrm{E}-01(=)$ & $2.220 \mathrm{E}-02(+)$ & \\
\hline & z-value (signedrank) & $-4.6774(164)$ & $-4.3399(200)$ & $-0.4499(615)$ & $0.4499(711)$ & $-2.2871(419)$ & \\
\hline & Mean (Mean_rank) & $1.00 \mathrm{E}+02(1)$ & $1.00 \mathrm{E}+02(1)$ & $1.00 \mathrm{E}+02(1)$ & $1.00 \mathrm{E}+02(1)$ & $2.821 \mathrm{E}+04(2)$ & $1.00 \mathrm{E}+02(1)$ \\
\hline & $S D$ & $1.385 \mathrm{E}-07$ & 8.716E-13 & $6.668 \mathrm{E}-10$ & $0.00 E+00$ & $7.551 \mathrm{E}+03$ & 3.565E-13 \\
\hline F27 & & & & & & & \\
\hline & p-value (h-value) & $5.145 \mathrm{E}-10(+)$ & $6.380 \mathrm{E}-02(=)$ & $5.145 \mathrm{E}-10(+)$ & $1.573 \mathrm{E}-01(=)$ & $5.145 \mathrm{E}-10(+)$ & \\
\hline & z-value (signedrank) & $-6.2146(0)$ & $-1.8537(10)$ & $-6.2146(0)$ & $-1.4142(0)$ & $-6.2146(0)$ & \\
\hline F28 & Mean (Mean_rank) & $1.00 E+02(1)$ & $1.00 \mathrm{E}+02(1)$ & $1.00 \mathrm{E}+02(1)$ & $1.00 E+02(1)$ & $1.282 \mathrm{E}+02(2)$ & $1.00 \mathrm{E}+02(1)$ \\
\hline
\end{tabular}




\begin{tabular}{|c|c|c|c|c|c|c|}
\hline$S D$ & $1.712 \mathrm{E}-10$ & $4.295 \mathrm{E}-13$ & $2.422 \mathrm{E}-10$ & 1.435E-13 & $7.414 \mathrm{E}+00$ & 1.435E-13 \\
\hline p-value (h-value) & 5.139E-10 (+) & 2.034E-07 (+) & 5.127E-10 (+) & $1.000 \mathrm{E}+00(=)$ & 5.145E-10 (+) & \\
\hline z-value (signedrank) & $-6.2148(0)$ & $-5.1962(0)$ & $-6.2152(0)$ & $-(0)$ & $-6.2146(0)$ & \\
\hline Average Mean_rank & 2.4667 & 2.5333 & 3.8000 & 3.0000 & 4.6667 & 1.7333 \\
\hline$+/=/-$ & $10 / 4 / 0$ & $7 / 5 / 3$ & $10 / 4 / 2$ & $7 / 5 / 3$ & 13/ 1/ 1 & \\
\hline
\end{tabular}

\section{Searching accuracy:}

From Table 2 and Table 3, we can conclude that SCAA performs the best searching accuracy as it outperforms its peers with a large margin in most of the problems. SCAA exhibits 11 best Mean values out of 13 for conventional problems (Table 2) and obtains 10 best Mean values out of 15 for CEC2015 functions (Table 3). Specifically, for the conventional problems, SCAA ranks the first on 5 multimodal problems (F8, F10-F13). These functions have multiple local optima around the global optimum, which can mislead the swarm into the non-optimal basin. While in SCAA, thanks to the proposed dynamic $\alpha$ adjustment strategy, it has successfully enabled the population to track more promising regions and realize the balance between exploration and exploitation on multimodal functions.

As for the CEC2015 functions, it is noticeable that all involved algorithms suffer from performance degradation and none of them can find the acceptable solutions. Even so, SCAA achieves most number of best Mean values compared with other algorithms. To be specific, the performance of SCAA is superior on the unimodal functions (F14 and F15), hybrid functions (F19-F21) and composition functions (F22-F28). However, SCAA performs mediocrely on the multimodal functions (F16-F18) in which SCAA ranks $2^{\text {th }}, 4^{\text {th }}$ and $3^{\text {th }}$ out of 6 algorithms on F16, F17 and F18, respectively. These functions possess a huge number of local optima [26] 
that are more complex than the conventional multimodal functions. The mediocre performance of SCAA may come from its $\alpha$ boundary constraint, which may block agents' jumping-out momentum to explore the whole search space. Therefore, this limitation can be further explored in the future work.

According to the statistic results in the last row of Table 2 and Table 3, the involved algorithms obtain different rank values on conventional problems and CEC2015 functions, which validate their distinguishing search performance. Nevertheless, SCAA has the minimum value in Average rank and obtains the first place of results in both tables, which confirm the superior searching accuracy of SCAA. In addition, SCAA performs relatively robust towards the 28 benchmark functions as evidenced by its smallest $S D$ values.

\section{Wilcoxon signed ranks test:}

From Tables 2 and 3, it is apparent that SCAA has significant better performance compared to its peers in most cases. For the conventional problems, SCAA are significantly better than GSA, MGSA- $\alpha$, FuzzyGSA, FS $\alpha$ (Increase) and FS $\alpha$ (Decrement) on 9, 9, 10, 6 and 10 functions, respectively. FS $\alpha$ (Increase) and FS $\alpha$ (Decrement) are statistically better than SCAA only on one function (F4), while GSA, MGSA- $\alpha$ and FuzzyGSA cannot statistically outperform SCAA on any conventional functions. As for the CEC2015 functions, when compared with GSA, MGSA- $\alpha$, FuzzyGSA, FS $\alpha$ (Increase) and FS $\alpha$ (Decrement), SCAA shows significantly better performance on 10, 7, 10, 7 and 13 functions and exhibits statistically worse performance just on 1, 3, 2, 3 and 1 functions, respectively. These statistic results have validated the competitive advantages of the proposed SCAA approach. 
Table 4

Convergence speed and reliability comparison among six algorithms on conventional functions at 30-D.

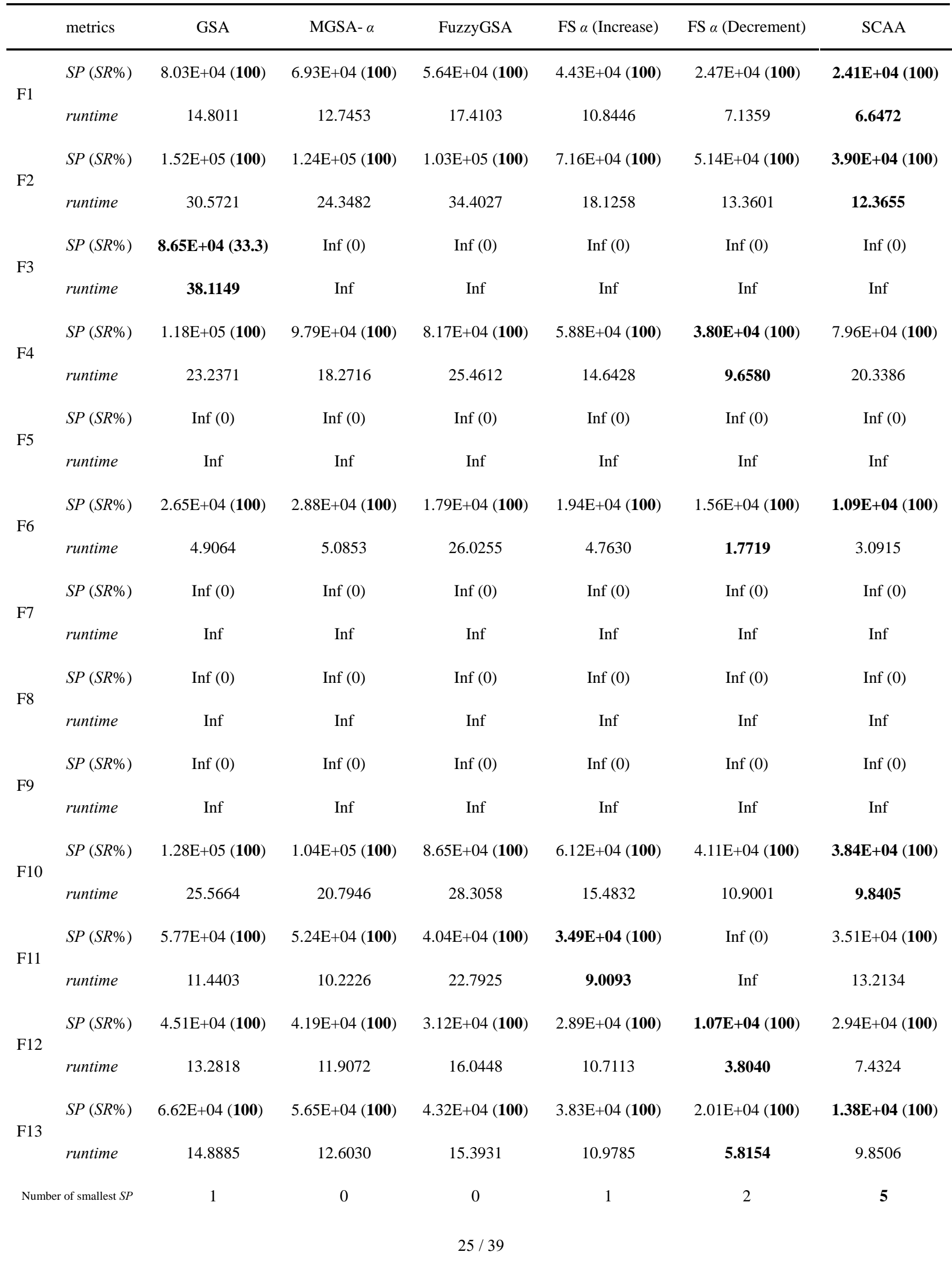




\section{Convergence comparison:}

The speed in obtaining an acceptable solution is also a salient yardstick for measuring the performance of an algorithm. For testing the searching efficiency of SCAA, the metric SP, runtime and convergence curves are also reported for comparison. It is worth mentioning that if one algorithm cannot solve the problem $(S R=0 \%)$, the $S P$ value is defined as infinity (Inf). Because of the strict threshold settings, none of the involved algorithms can achieve the available $S P$ values on all CEC2015 functions. Thus, only the results on 13 conventional functions are recorded. As shown in Table 4, SCAA achieves the smallest $S P$ values on 5 conventional problems (F1, F2, F6, F10 and F13), which is obviously better than that of its 5 peers. The fast convergence characteristic of SCAA mainly results from its adaptive adjustment of the parameter alpha.

As for the runtime results, SCAA has a tendency to spend slightly more computational time due to its repeating calculation of the ratio of mass and distance between any two agents in the stability conditions in each iteration. Nevertheless, from Table 4 we can conclude that SCAA spends the least computational time on unimodal functions F1 and F2. These results may benefit from the superior $S P$ results of SCAA, which can decrease its runtime values to some extent. For more complicated functions, superiority on efficiency of SCAA is not as obvious as that on F1 and F2. This is mainly because that the global optimum in these functions can be very far away from the local optima or can be surrounded by a considerable amount of local optima [4]. In this situation, for avoiding trapping into the local optima, the parameter alpha in SCAA is adjusted to decrease the convergence tendency of agents to the elite masses, which causes the increase of execution time. Even so, the average runtime of SCAA ranks the 
second among the competing algorithms as listed at the bottom of Table 4. In this experiment, FS $\alpha$ (Decrement) ranks the first according to its average runtime. This is primarily because the decreasing trend of $\alpha$ from an initial value 150 enables FS $\alpha$ (Decrement) to have a much larger $\alpha$ value during the whole iteration, which can improve the convergence speed. However, the larger alpha value can lead to the smaller search steps of agents and easily cause the premature convergence. This is observed by the poor searching performance of FS $\alpha$ (Decrement) as shown in Table 2 of the manuscript.

A closer look at the convergence curves of different algorithms in Fig. 3 provides more insight into their searching behavior. The figure includes the representative conventional problems (F1, F2, F3, F5, F10 and F13) and CEC2015 functions (F14, F19, F20, F21, F23 and F24). Based on the graphical results in Fig. 3, the outstanding convergence characteristics of SCAA on different test functions are explained. Specifically, for the conventional problems, the convergence curves of SCAA sharply drop at one point on functions F1, F2, F3, F5, F10 and F13 in the early iterations and then reach better results in the latter iterations. These observations prove the capability of SCAA to facilitate the balance between exploration and exploitation. With regard to the CEC2015 functions, because of their complex characteristics, the convergence speeds of all involved algorithms are slightly inferior to that in conventional problems. Nevertheless, the convergence curves of SCAA rapidly drop at one point on functions F14, F19, F23 and F24 in the early iterations. On functions F20 and F21, the convergence speed of SCAA is more slowly than its peers at the early stage, while its speed increases in the middle stage of optimization. Moreover, SCAA achieves superior convergence accuracy among all algorithms, which may result from the ability of $\alpha$ boundary constraint to improve convergence precision in the latter iterations. In general, SCAA has produced improved searching efficiency compared with other algorithms. 

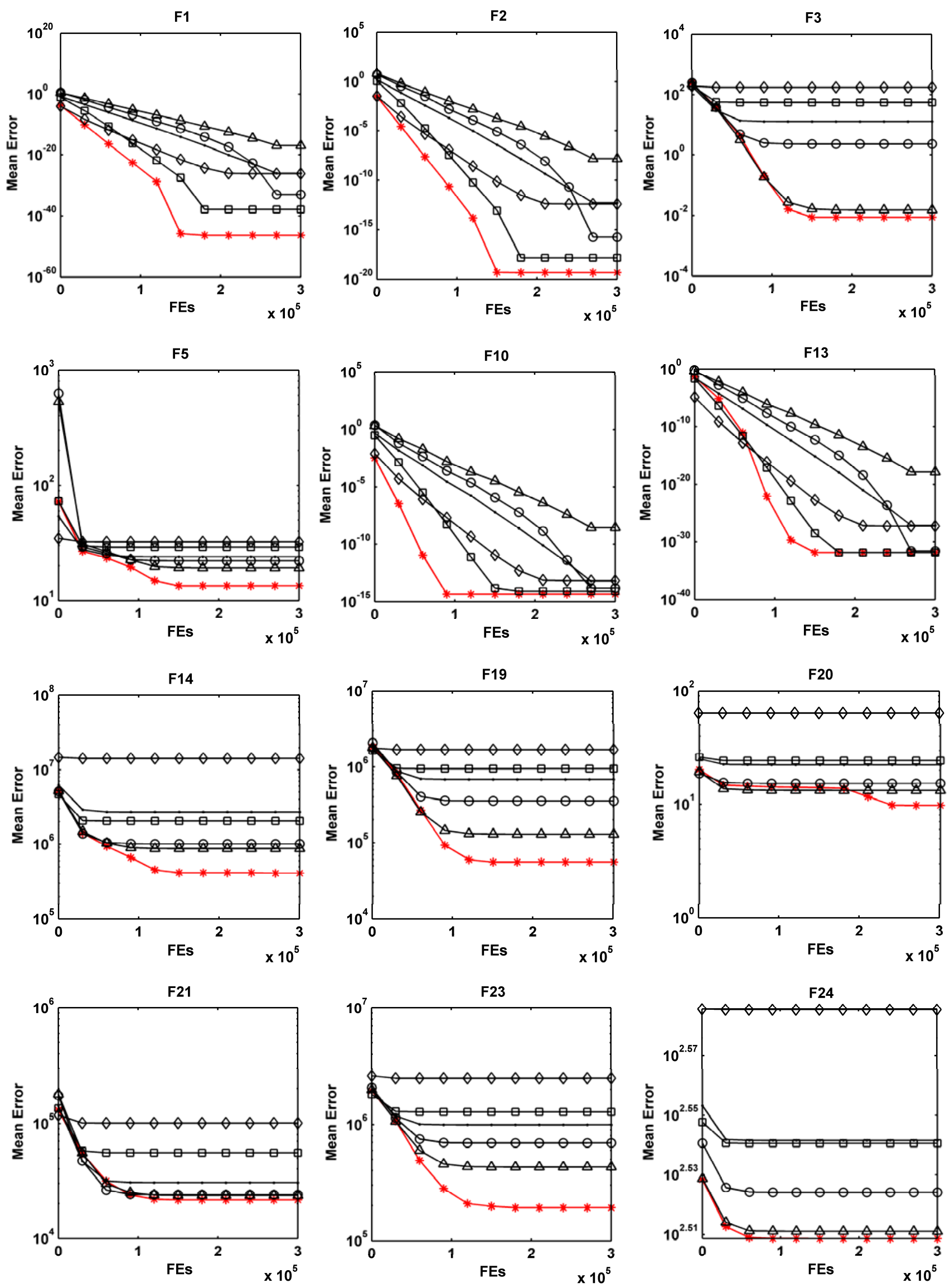

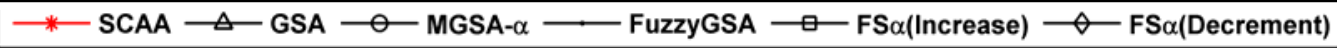


Fig. 3. Convergence curves of different alpha adjusting GSA variants.

\subsection{Stability analysis of SCAA}

In order to validate the stability of SCAA, the trajectories of agents are analyzed. Experiments are conducted on three conventional functions F2, F7 and F9, respectively. For a better observation, the number of iterations is set to 100 and the trajectory of the first dimension for the first agent, denoted as $x_{11}$ in each algorithm, is recorded. Experiments are carried out with 51 independent runs and the average trajectory curves are listed in Fig. 4. Note that the stability stage occurs when there is little variation in an agent's position between iterations $[8,18]$.

First, in order to analyze the effect of stability boundary constraint, we compare the performance of SCAA in two cases: SCAA with stability constraints and SCAA without stability constraints (denoted as SCAA-Ns). From the trajectory curves in Fig. 4 (a)-(c), it is clear that the agent's trajectory in red line (SCAA) are more stable than that in blue line (SCAA-Ns), which validates that the stability constraints play an important role in guaranteeing the stable convergence.

Second, we make the stability comparison between SCAA and other involved algorithms. The results are plotted in Fig. 4 (d)-(f). It can be observed that SCAA performs more stable when compared with other algorithms. To be specific, the agent trajectories of GSA, FS $\alpha$ (Increase) and MGSA- $\alpha$ are rather unstable, whereas SCAA and FS $\alpha$ (Decrement) are more stable. Particularly, the agent trajectories of FS $\alpha$ (Decrement) is more stable than that in SCAA. This may because that the decreasing trend of $\alpha$ from an initial value 150 in FS $\alpha$ (Decrement) 


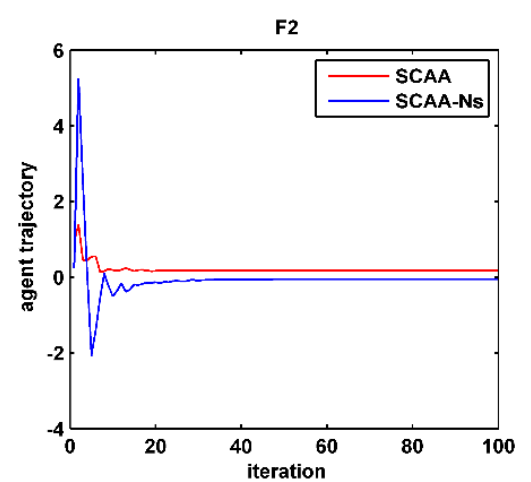

(a) F2

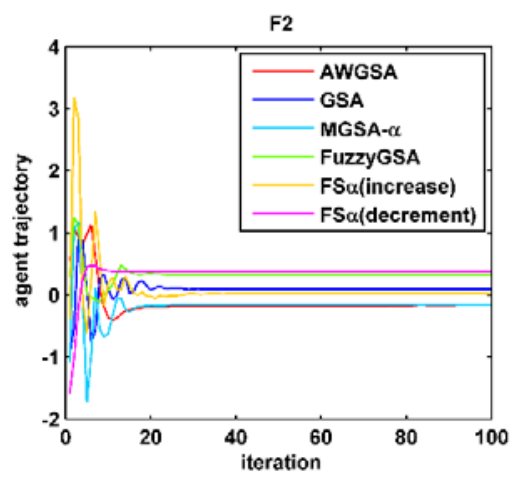

(d) F2

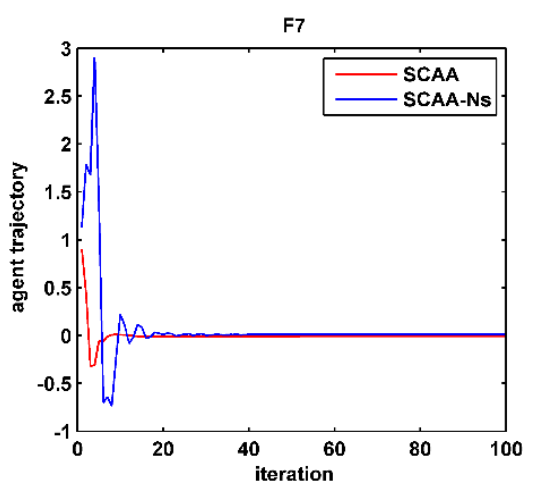

(b) F7

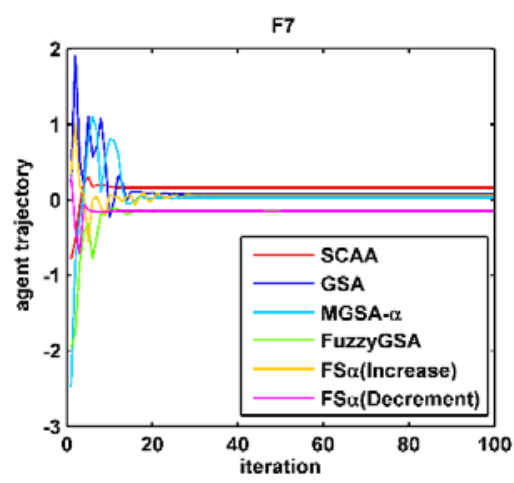

(e) F7

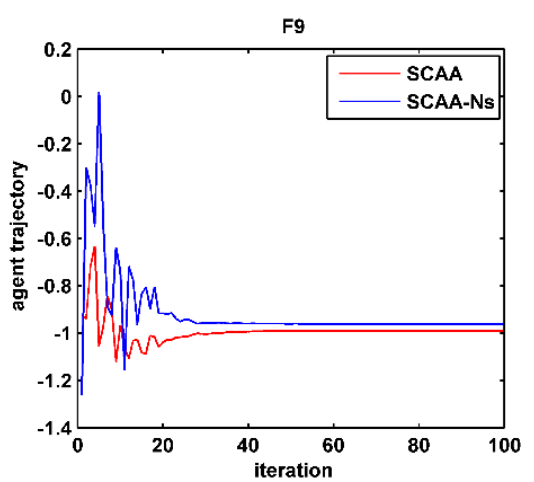

(c) F9

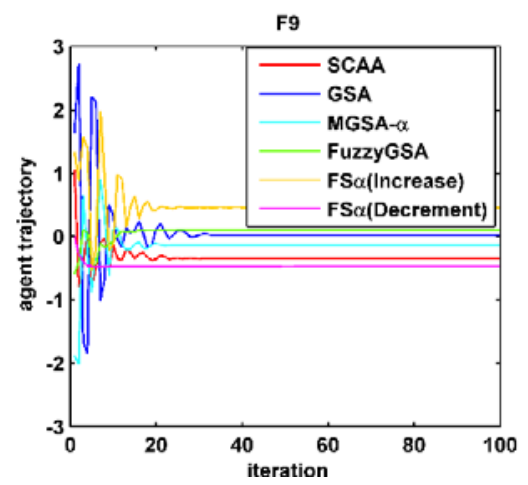

(f) F9

Fig. 4. Agent trajectories of different algorithms.

The $l p$ and $\alpha_{\max }$ are two key parameters that affect the searching performance of SCAA. To evaluate the impact of $l p$ and $\alpha_{\max }$ on different kinds of functions, we conduct parameter sensitivity analysis on 30 dimensional versions of the 14 representative functions including 4 unimodal conventional functions (F1, F2, F3, 
F5), 4 multimodal conventional functions (F9, F10, F12, F13) and 6 rotated and shifted functions (F14, F19, F20, F23-F25) in this study.

We first carried out experiments with $l p=[1,2,3,4,5,6,7,8,9,10]$ with all other parameters set as discussed

in Section 4.1. Then we perform sensitivity analysis experiment of parameter $\alpha_{\max }$ with $\alpha_{\max }=[40,50,60,70,80$,

(1) Sensitivity analysis of the parameter $l p$
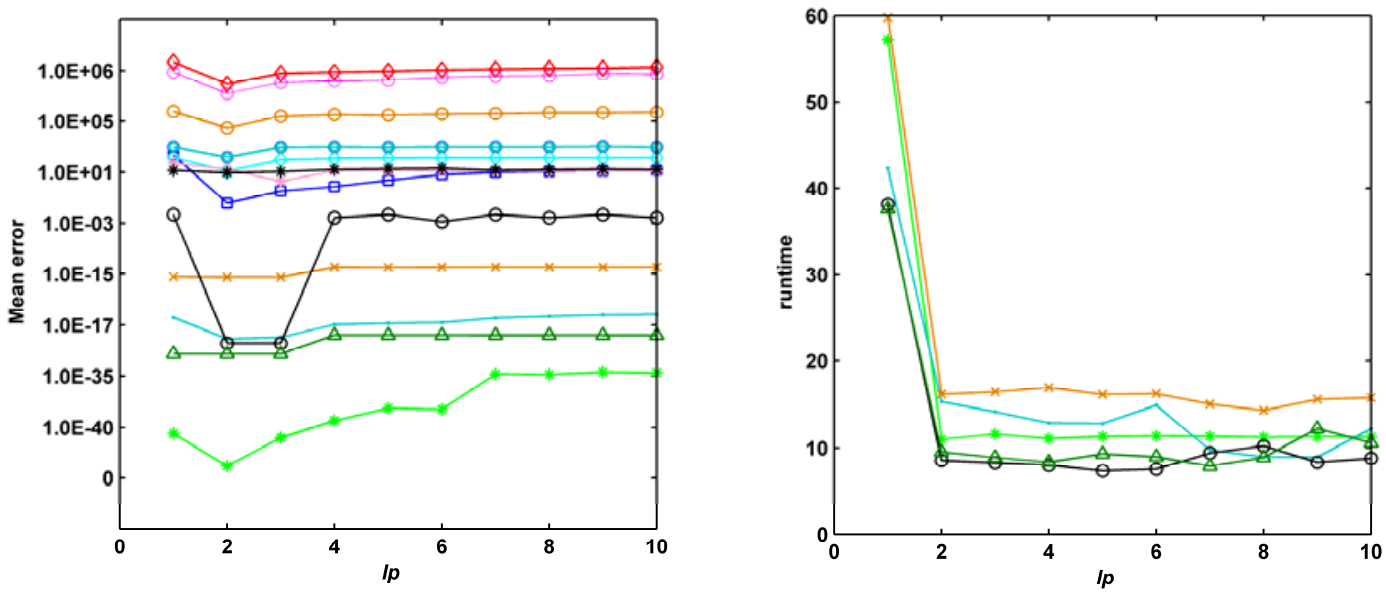

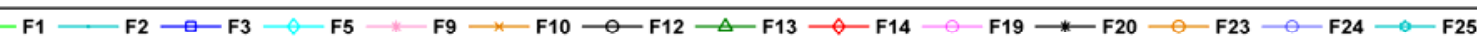

(a) The Mean values of SCAA with different $l p$

(b) The runtime values of SCAA with different $l p$ 
Fig. 5. Effects of $l p$ on the performance of SCAA on conventional functions at 30- $D$. (The vertical axis of (a) is not to scale for easy presentation of the data).

In SCAA, the parameter $l p$ determines the frequency of alpha changing. A suitable $l p$ can activate the alpha changing of stagnated agents without wasting too much computational cost. A too small $l p$ value may cause agent's oscillation and disturb the swarm convergence, while a too large value for $l p$ will result in the waste of computation on the local optima and lead to a premature convergence.

From Fig. 5, it is apparent that the search accuracy (Mean) and efficiency (runtime) of SCAA are affected by the parameter $l p$. As shown in Fig. 5 (a), SCAA achieves the best Mean results when $l p=2$ on unimodal conventional functions. This is mainly owing to the fact that these functions have only one minimum in the search space. Thus, a small $l p$ can make agents quickly adjust the search direction to detect the promising regions and then rapidly enlarge the alpha value to emphasize exploitation around good solutions. With respect to the multimodal conventional functions, the setting $l p=3$ is most suitable for SCAA as shown in Fig. 5 (a). This is probably because that a small $l p$ may lead to the oscillation of agents in local optima and limit the probabilities to find other promising areas. Thus, on the multimodal functions, a relatively larger setting $l p=3$ is recommended in this paper. For the rotated and shifted functions, SCAA performs the best searching accuracy when $l p=2$. These functions are more complex than the conventional functions, where most of them are asymmetrical and have different properties around different local optima [26]. In this situation, a small $l p$ can sufficiently monitor the problem environment and quickly change the search behavior of agents. 
In terms of the runtime as shown in Fig. 5 (b), it is obvious that the execution time of SCAA is significantly reduced when $l p$ is increased from 1 to 2 for most testing functions. When the $l p$ increases from 2 to 10 , the differences of runtime on each function is minor. Considering that the search accuracy of SCAA becomes worse when $l p \geq 6$ as shown in Fig. 5 (a), we recommend $l p=2,3$ and 2 for the unimodal conventional functions, multimodal conventional functions, rotated and shifted functions respectively in this paper.
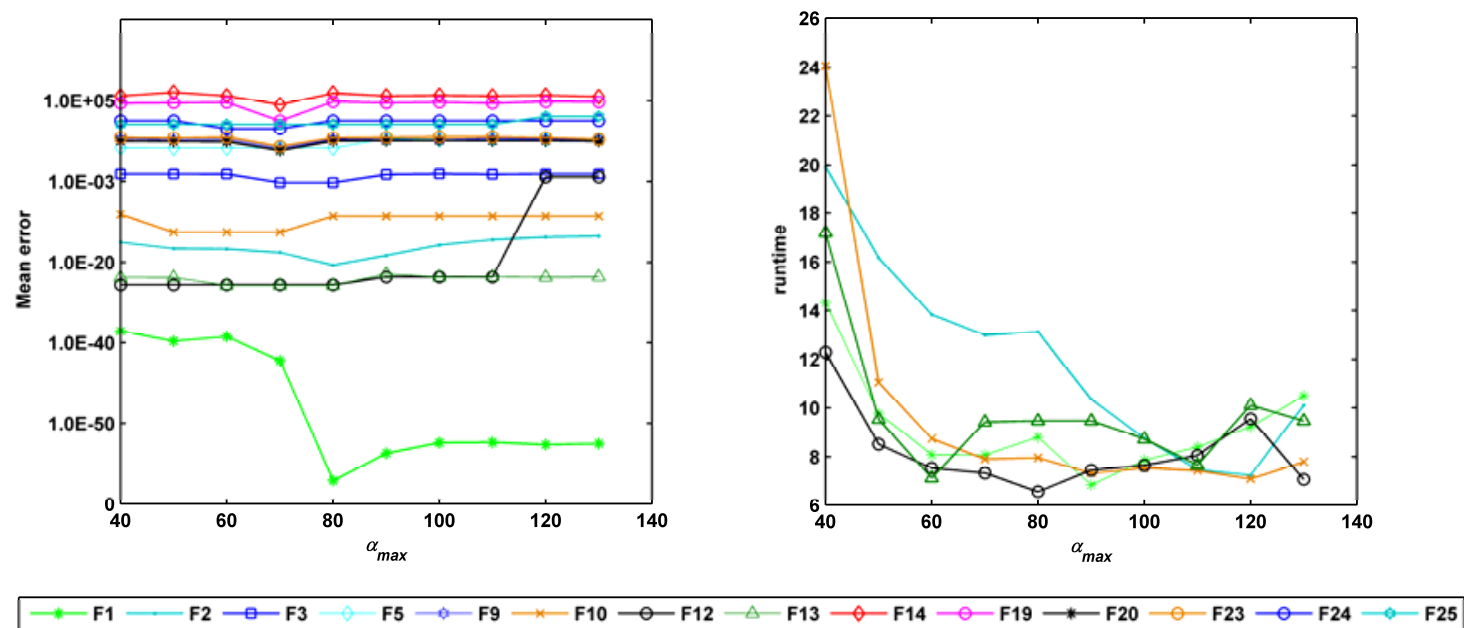

(a) The Mean values of SCAA with different $\alpha_{\max }$

(b) The runtime values of SCAA with different $\alpha_{\max }$

Fig. 6. Effects of $\alpha_{\max }$ on the performance of SCAA on conventional functions at 30- $D$. (The vertical axis of (a) is not to scale for easy presentation of the data).

From Fig. 6, it can be seen that the performance of SCAA is also affected by the parameter $\alpha_{\max }$. A smaller $\alpha_{\max }$ may slow down the convergence of SCAA while a larger one may lead to the searching stagnation and prematurity problem. As plotted in Fig. 6 (a), the searching accuracy of SCAA is the best when $\alpha_{\max }=80$ on unimodal conventional functions. This is mainly due to that a larger $\alpha_{\max }$ can enhance the hill-climbing 
performance on functions with only one minimum. When SCAA is applied to solve the multimodal functions and the rotated and shifted functions, the setting $\alpha_{\max }=70$ becomes the best choice. These functions have lots of minimum and are more complex than the unimodal functions. Thereby a smaller values of $\alpha_{\max }$ may ensure the global search abilities of agents and enables them to escape from the local optima.

As for the runtime results, it is apparent that the execution time of SCAA is dramatically reduced in most cases when $\alpha_{\max }$ is increased from 40 to 50 . When $\alpha_{\max } \geq 50$, the differences of runtime on each function is minor. These runtime distribution results support the analysis that a larger $\alpha_{\max }$ is beneficial for the convergence speed. However, if the value of $\alpha_{\max }$ becomes too large, it will lead to high Mean errors as reported in Fig. 6 (a). Considering both the search accuracy and efficiency of SCAA, we set $\alpha_{\max }$ to 80,70 and 70 for unimodal conventional functions, multimodal conventional functions, rotated and shifted functions, respectively in this paper.

\section{Conclusion and further study}

In this paper, we proposed a stability constrained adaptive alpha for GSA (SCAA) to enhance the search performance of the original GSA. In SCAA, the evolutionary state of each agent was first estimated. Then, the variation of an agent's position and fitness is employed as feedback to guide the adjustment of $\alpha$ according to its current state. This adaptive alpha adjusting strategy can enhance the convergence when an agent finds a promising direction and relieves the premature problem when an agent moves to a local optimum. In addition, a boundary 
constraint derived from the stability conditions was put forward to restrict agents' $\alpha$ values in each iteration, which has guaranteed agents’ stable trajectories and improved the precision of convergence.

To verify the performance of SCAA, 28 benchmark functions including conventional problems and CEC2015 functions were tested in this paper. Simulation results and comparisons have clearly showed the superiority of the proposed SCAA over the original GSA and other alpha adjusting algorithms in terms of the searching accuracy, searching reliability and searching efficiency. Besides, the stability analysis has demonstrated the effect of $\alpha$ boundary constraint and validated the stability of SCAA.

One area in which SCAA falls short is the constant setting of the alpha upper boundary $\alpha_{\max }$, which lacks the time-varying characteristics as the lower boundary does. This reveals an area where SCAA could be further improved in the future work. Another potential direction for improvement is to consider the adaptive information systems, which have received increasing attention and are widely used for different applications, such as customer churn prediction [29], cloud computing [16], case-based reasoning [30] and clustering of uncertain data [28]. A number of adaptive metaheuristics techniques have been applied to control key parameters for improving the optimization performance. For example, in [38,51], an improved version of the teaching-learning-based optimization (TLBO) algorithm was proposed, in which an adaptive teaching factor was considered. Similarly, in [41], a new variant of TLBO was presented by integrating a self-adaptive strategy for population sizing. Besides, Tejani et al. [50] introduced adaptive benefit factors into the symbiotic organisms search (SOS) to enhance its searching efficiency. Jia et al. [15] put forward an improved cuckoo search (ICS) algorithm by employing adaptive technique in the step length of levy flight and discovering probability. R Sridhar proposed an adaptive genetic algorithm to optimize the bin packing problem [44]. How to combine GSA with adaptive systems may have great 
potential to further improve the optimization performance, where SCAA can also be applied to more real-world optimization problems.

\section{Acknowledgements}

This work was supported by Chinese Natural Science Foundation Projects (No. 41471353), Fundamental Research Funds for the Central Universities (No. 15CX06001A).

\section{References}

[1] H. Askari, S.-H. Zahiri, Decision function estimation using intelligent gravitational search algorithm, Int. J. Mach. Learn. Cybern. 3 (2) (2012) 163-172, http://dx.doi.org/10.1007/s13042-011-0052-x.

[2] M.S.I.B. Aziz, S.W. Nawawi, S. Sudin, N. Abd Wahab, Exploitation selection of alpha parameter in Gravitational Search Algorithm of PID controller for computational time analysis, in: Control System, Computing and Engineering (ICCSCE), 2014 IEEE International Conference on, IEEE, 2014, pp. 112-117, http://dx.doi.org/10.1109/ICCSCE.2014.7072699.

[3] T. Back, Evolutionary algorithms in theory and practice: evolution strategies, evolutionary programming, genetic algorithms, Oxford university press, 1996, http://dx.doi.org/10.1108/k.1998.27.8.979.4.

[4] Z. Chen, X. Yuan, H. Tian, B. Ji, Improved gravitational search algorithm for parameter identification of water turbine regulation system, Energy Conversion \& Management $78 \quad$ (30) (2014) 306-315, http://doi.org/10.1016/j.enconman.2013.10.060.

[5] P. Civicioglu, Artificial cooperative search algorithm for numerical optimization problems, Inf. Sci. 229 (6) (2013) 58-76, http://dx.doi.org/10.1016/j.ins.2012.11.013.

[6] J. Derrac, S. García, D. Molina, F. Herrera, A practical tutorial on the use of nonparametric statistical tests as a methodology for comparing evolutionary and swarm intelligence algorithms, Swarm Evol. Comput. 1 (1) (2011) 3-18, http://dx.doi.org/10.1016/j.swevo.2011.02.002.

[7] M. Doraghinejad, H. Nezamabadi-Pour, Black hole: A new operator for gravitational search algorithm, Int. J. Comput. Intell. Syst. 7 (5) (2014) 809-826, http://dx.doi.org/10.1080/18756891.2014.966990.

[8] F. Farivar, M.A. Shoorehdeli, Stability analysis of particle dynamics in gravitational search optimization algorithm, Inf. Sci. 337 (2016) 25-43, http://dx.doi.org/10.1016/j.ins.2015.12.017. 
[9] S. Gao, C. Vairappan, Y. Wang, Q. Cao, Z. Tang, Gravitational search algorithm combined with chaos for unconstrained numerical optimization, Appl. Math. Comput. 231 (2014) 48-62, http://dx.doi.org/10.1016/j.amc.2013.12.175.

[10] S. García, D. Molina, M. Lozano, F. Herrera, A study on the use of non-parametric tests for analyzing the evolutionary algorithms' behaviour: a case study on the CEC'2005 Special Session on Real Parameter Optimization, J. Heuristics. 15 (6) (2008) 617-644, http://dx.doi.org/10.1007/s10732-008-9080-4.

[11] B. González, F. Valdez, P. Melin, G. Prado-Arechiga, Fuzzy logic in the gravitational search algorithm for the optimization of modular neural networks in pattern recognition, Expert. Syst. Appl. 42 (14) (2015) 5839-5847, http://dx.doi.org/10.1016/j.eswa.2015.03.034.

[12] Z. Guo, A Hybrid Optimization Algorithm Based on Artificial Bee Colony and Gravitational Search Algorithm, International Journal of Digital Content Technology \& Its Applications 6 (17) (2012) 620-626, http://doi.org/10.4156/jdcta.vol6.issue17.68.

[13] X.H. Han, X.M. Chang, L. Quan, X.Y. Xiong, J.X. Li, et al, Feature subset selection by gravitational search algorithm optimization, Inf. Sci. 281 (2014) 128-146, http://doi.org/10.1016/j.ins.2014.05.030.

[14] X.H. Han, L. Quan, X.Y. Xiong, B. Wu, Facing the classification of binary problems with a hybrid system based on quantum-inspired binary gravitational search algorithm and K-NN method, Eng. Appl. Artif. Intel 26 (10) (2013) 2424-2430, http://doi.org/10.1016/j.engappai.2013.05.011.

[15] B. Jia, B. Yu, Q. Wu, C. Wei, R. Law, Adaptive affinity propagation method based on improved cuckoo search, Knowl-based. Syst. 111 (C) (2016) 27-35, http://dx.doi.org/10.1016/j.knosys.2016.07.039.

[16] J. Jiang, J. Lu, G. Zhang, An Innovative Self-Adaptive Configuration Optimization System in Cloud Computing, in: IEEE Ninth International Conference on Dependable, Autonomic and Secure Computing, 2011, pp. 621-627, http://dx.doi.org/10.1109/dasc.2011.112.

[17] S. Jiang, Z. Ji, Y. Shen, A novel hybrid particle swarm optimization and gravitational search algorithm for solving economic emission load dispatch problems with various practical constraints, Int. J. Elec. Power 55 (2) (2014) 628-644, http://dx.doi.org/10.1016/j.ijepes.2013.10.006.

[18] S. Jiang, Y. Wang, Z. Ji, Convergence analysis and performance of an improved gravitational search algorithm, Appl. Math. Comput. 24 (2014) 363-384, http://dx.doi.org/10.1016/j.asoc.2014.07.016.

[19] D. Karaboga, B. Basturk, Artificial bee colony (ABC) optimization algorithm for solving constrained optimization problems, in: International Fuzzy Systems Association World Congress, Springer, Berlin/Heidelberg, 2007, pp. 789-798, http://dx.doi.org/10.1007/978-3-540-72950-1_77.

[20] M. Khajehzadeh, M.R. Taha, A. El-Shafie, M. Eslami, A modified gravitational search algorithm for slope stability analysis, Eng. Appl. Artif. Intel 25 (8) (2012) 1589-1597, http://dx.doi.org/10.1016/j.engappai.2012.01.011.

[21] S.U. Khan, S. Yang, L. Wang, L. Liu, A Modified Particle Swarm Optimization Algorithm for Global Optimizations of Inverse Problems, IEEE Transactions on Magnetics 52 (3) (2016) 1-4, http://dx.doi.org/10.1109/TMAG.2015.2487678.

[22] C. Li, L. Chang, Z. Huang, Y. Liu, N. Zhang, Parameter identification of a nonlinear model of hydraulic turbine governing system with an elastic water hammer based on a modified gravitational search algorithm, Eng. Appl. Artif. Intel 50 (2016) 177-191, http://dx.doi.org/10.1016/j.engappai.2015.12.016.

[23] C. Li, H. Li, P. Kou, Piecewise function based gravitational search algorithm and its application on parameter identification of AVR system, Neurocomputing $124 \quad$ (2014) 139-148, http://dx.doi.org/10.1016/j.neucom.2013.07.018.

[24] X. Li, J. Wang, J. Zhou, M. Yin, An effective GSA based memetic algorithm for permutation flow shop scheduling, in: Evolutionary Computation, 2010, pp. 1-6, http://doi.org/10.1109/cec.2010.5586392 
[25] X. Li, M. Yin, Z. Ma, X. Li, Z. Ma, Hybrid differential evolution and gravitation search algorithm for unconstrained optimization, Int. J. Phys. Sci. 6 (25) (2011) 5961-5981, http://dx.doi.org/10.5897/IJPS11.029.

[26] J. Liang, B. Qu, P. Suganthan, Q. Chen, Problem definition and evaluation criteria for the CEC 2015 competition on learning-based real-parameter single objective optimization (2014), Computational Intelligence Laboratory, Zhengzhou University, Zhengzhou China and Technical Report, Nanyang Technological University, Singapore (2014).

[27] W.H. Lim, N.A. Mat Isa, Teaching and peer-learning particle swarm optimization, Appl. Math. Comput. 18 (2014) 39-58, http://dx.doi.org/10.1016/j.asoc.2014.01.009.

[28] H. Liu, X. Zhang, X. Zhang, Y. Cui, Self-adapted mixture distance measure for clustering uncertain data, Knowl-based. Syst. (2017) http://dx.doi.org/10.1016/j.knosys.2017.04.002.

[29] N. Lu, H. Lin, J. Lu, G. Zhang, A Customer Churn Prediction Model in Telecom Industry Using Boosting, IEEE Transactions on Industrial Informatics 10 (2) (2014) 1659-1665, http://dx.doi.org/10.1109/tii.2012.2224355.

[30] N. Lu, J. Lu, G. Zhang, An Integrated Knowledge Adaption Framework for Case-Based Reasoning Systems, in: International Conference on Knowledge-Based and Intelligent Information and Engineering Systems, 2009, pp. 372-379.

[31] S. Mirjalili, S.Z.M. Hashim, A new hybrid PSOGSA algorithm for function optimization, in: Computer and Information Application (ICCIA), 2010 International Conference on, 2010, pp. 374-377, http://dx.doi.org/10.1109/ICCIA.2010.6141614.

[32] S. Mirjalili, A. Lewis, Adaptive gbest-guided gravitational search algorithm, Neural. Comput. Appl. 25 (7-8) (2014) 1569-1584, http://dx.doi.org/10.1007/s00521-014-1640-y.

[33] H. Nezamabadi-Pour, F. Barani, Gravitational Search Algorithm: Concepts, Variants, and Operators, Modern Optimization Algorithms and Applications in Engineering and Economics (2016).

[34] R. Poli, J. Kennedy, T. Blackwell, Particle swarm optimization, Swarm intelligence 1 (1) (2007) 33-57, http://dx.doi.org/10.1007/s11721-007-0002-0.

[35] E. Rashedi, H. Nezamabadi-Pour, S. Saryazdi, GSA: A Gravitational Search Algorithm, Inf. Sci. 179 (13) (2009) 2232-2248, http://dx.doi.org/10.1016/j.ins.2009.03.004.

[36] F. Saeidi-Khabisi, E. Rashedi, Fuzzy gravitational search algorithm, in: Proceedings 2nd international econference on computer and knowledge engineering (ICCKE), 2012, pp. 156-160, http://dx.doi.org/10.1109/iccke.2012.6395370

[37] S. Sarafrazi, H. Nezamabadi-Pour, S. Saryazdi, Disruption: A new operator in gravitational search algorithm, Sci Iranica 18 (3) (2011) 539-548, http://dx.doi.org/10.1016/j.scient.2011.04.003.

[38] V.J. Savsani, G.G. Tejani, V.K. Patel, Truss topology optimization with static and dynamic constraints using modified subpopulation teaching-learning-based optimization, Eng. Optimiz. $48 \quad$ (11) (2016) 1-17, http://dx.doi.org/10.1080/0305215x.2016.1150468.

[39] S. Sheikhpour, M. Sabouri, S.H. Zahiri, A hybrid Gravitational search algorithm — Genetic algorithm for neural network training, in: Electr. Eng., 2013, pp. 1-5, http://dx.doi.org/10.1109/iraniancee.2013.6599894.

[40] N. Siddique, H. Adeli, Gravitational Search Algorithm and Its Variants, International Journal of Pattern Recognition \& Artificial Intelligence (2015) http://dx.doi.org/10.1142/S0218001416390018.

[41] S. Sleesongsom, S. Bureerat, Four-Bar Linkage Path Generation through Self-Adaptive Population Size Teaching-Learning Based Optimization, Knowl-based. Syst. (2017) http://dx.doi.org/10.1016/j.knosys.2017.08.012.

[42] M. Soleimanpour-Moghadam, H. Nezamabadi-Pour, An improved quantum behaved gravitational search algorithm, in: Electr. Eng., 2012, pp. 711-715, http://dx.doi.org/10.1109/iraniancee.2012.6292446.

[43] A. Sombra, F. Valdez, P. Melin, O. Castillo, A new gravitational search algorithm using fuzzy logic to parameter adaptation, in: Evolutionary Computation (CEC), 2013 IEEE Congress on, IEEE, 2013, pp. 1068-1074, http://dx.doi.org/10.1109/CEC.2013.6557685. 
[44] R. Sridhar, M. Chandrasekaran, C. Sriramya, T. Page, Optimization of heterogeneous Bin packing using adaptive genetic algorithm, (2017) 012026, http://dx.doi.org/10.1088/1757-899x/183/1/012026.

[45] R. Storn, K. Price, Differential evolution-a simple and efficient adaptive scheme for global optimization over continuous spaces, ICSI Berkeley, 1995, http://dx.doi.org/10.1063/1.4930637.

[46] P.N. Suganthan, N. Hansen, J.J. Liang, K. Deb, Y.P. Chen, et al, Problem Definitions and Evaluation Criteria for the CEC 2005 Special Session on Real-Parameter Optimization, Nanyang Technological University (2005).

[47] G. Sun, A. Zhang, A Hybrid Genetic Algorithm and Gravitational Search Algorithm for Image Segmentation Using Multilevel Thresholding, in: Iberian Conference on Pattern Recognition and Image Analysis, Springer, Berlin Heidelberg, 2013, pp. 707-714, http://dx.doi.org/10.1007/978-3-642-38628-2 84.

[48] G. Sun, A. Zhang, Z. Wang, Y. Yao, J. Ma, G.D. Couples, Locally informed Gravitational Search Algorithm, Knowl-based. Syst. 104 (2016) 134-144, http://dx.doi.org/10.1016/j.knosys.2016.04.017.

[49] M. Taherkhani, R. Safabakhsh, A novel stability-based adaptive inertia weight for particle swarm optimization, Appl. Math. Comput. 38 (2016) 281-295, http://dx.doi.org/10.1016/j.asoc.2015.10.004.

[50] G.G. Tejani, V.J. Savsani, V.K. Patel, Adaptive symbiotic organisms search (SOS) algorithm for structural design optimization, Journal of Computational Design \& Engineering 3 (3) (2016) 226-249, http://dx.doi.org/10.1016/j.jcde.2016.02.003.

[51] G.G. Tejani, V.J. Savsani, V.K. Patel, Modified sub-population teaching-learning-based optimization for design of truss structures with natural frequency constraints, Mechanics Based Design of Structures \& Machines 44 (7) (2016) págs. 495-513, http://dx.doi.org/10.1080/15397734.2015.1124023.

[52] J. Vijaya Kumar, D.M. Vinod Kumar, K. Edukondalu, Strategic bidding using fuzzy adaptive gravitational search algorithm in a pool based electricity market, Appl. Math. Comput. 13 (5) (2013) 2445-2455, http://dx.doi.org/10.1016/j.asoc.2012.12.003.

[53] J. Xiao, F. Qi, Y. Li, Gravitational chaotic search algorithm for partners selection with due date constraint in virtual enterprise, in: Advanced Computational Intelligence (IWACI), 2011 Fourth International Workshop on, IEEE, 2011, pp. 138-142, http://dx.doi.org/10.1109/iwaci.2011.6159990.

[54] X. Yao, Y. Liu, G. Lin, Evolutionary programming made faster, Ieee. T. Evolut. Comput. 3 (2) (1999) 82-102, http://dx.doi.org/10.1109/4235.771163.

[55] A. Zhang, G. Sun, J. Ren, X. Li, Z. Wang, X. Jia, A Dynamic Neighborhood Learning-Based Gravitational Search Algorithm, IEEE Transactions on Cybernetics PP (99) (2016) 1-12.

[56] N. Zhang, C. Li, R. Li, X. Lai, Y. Zhang, A mixed-strategy based gravitational search algorithm for parameter identification of hydraulic turbine governing system, Knowl-based. Syst. (2016) http://dx.doi.org/10.1016/j.knosys.2016.07.005. 